\title{
NONLINEAR STABILIZING CONTROL OF AN UNCERTAIN BIOPROCESS MODEL
}

\author{
Neli DiMitRoVA, Mikhail KRASTANOV \\ Department of Biomathematics, Institute of Mathematics and Informatics \\ Bulgarian Academy of Sciences, Acad. G. Bonchev str., bl. 8, 1113 Sofia, Bulgaria \\ e-mail: nelid@bio.bas.bg, krast@math.bas.bg
}

\begin{abstract}
In this paper we consider a nonlinear model of a biological wastewater treatment process, based on two microbial populations and two substrates. The model, described by a four-dimensional dynamic system, is known to be practically verified and reliable. First we study the equilibrium points of the open-loop system, their stability and local bifurcations with respect to the control variable. Further we propose a feedback control law for asymptotic stabilization of the closed-loop system towards a previously chosen operating point. A numerical extremum seeking algorithm is designed to stabilize the dynamics towards the maximum methane output flow rate in the presence of coefficient uncertainties. Computer simulations in Maple are reported to illustrate the theoretical results.
\end{abstract}

Keywords: wastewater treatment model, local bifurcations of steady states, asymptotic stabilization, extremum seeking, uncertain data.

\section{Introduction}

Biological wastewater treatment using anaerobic digestion is a process where microorganisms decompose the organic compounds inside the effluent to reduce the pollutant concentration in the outlet stream below a specified value, usually fixed by environmental and safety rules. At the same time, this process can also produce valuable energy (methane). This complex ecosystem involves plenty of bacterial species, whose dynamics are difficult to grasp due to physiological reasons. The operation of such processes poses a number of practical problems, since anaerobic digestion is a complex nonlinear system, which is known to be unstable, see, e.g., (Antonelli et al., 2003) and the references there. One of the main drawbacks in the modelling and control of anaerobic digestion lies in the difficulty to monitor on-line the key biological variables of the process. A second drawback is the high uncertainty of the models describing the biological process; this is due to the fact that in most cases the reproducibility of laboratory and practical experiments is not guaranteed, hence the experimental data are noisy and the reason for the noise is difficult to specify. Thus developing control systems only based on simple measurements that guarantee the stability of the process in the presence of uncertainties is of primary importance. For further information about different control approaches see, e.g., (Antonelli et al., 2003; Heinzle et al., 1993; Mailert et al., 2004) and the references there.

The present paper is devoted to studying a fourdimensional nonlinear control system that models a wastewater treatment process. In the previous work (Dimitrova and Krastanov, 2006), the authors designed an adaptive stabilizing feedback control law for the same model in the presence of parameter uncertainties. This adaptive feedback depends on observable state variables $s_{1}$ and $x_{1}$ (see the definitions below) and stabilizes asymptotically the closed-loop system towards an (unknown) equilibrium point such that its projection on the $s_{1}$-axis is equal to a previously chosen operating point $s_{1}^{*}$. Here we propose a feedback law for asymptotic stabilization that is more practically oriented, i.e., it depends only on on-line measurable quantities.

The paper is organized as follows: Section 2 presents shortly the dynamic model of the biological wastewater treatment process. Section 3 is devoted to studying the local one-parameter bifurcations of the equilibrium points of the open-loop system: it is shown that the dynamics undergo transcritical bifurcations with respect to the control input (the dilution rate) $u$. Assuming that the model parameters are unknown but bounded within intervals, asymptotic stabilization of the dynamic system towards 
a previously chosen operating point (called also reference or set point) is studied in Section 4. In order to prove that the closed-loop system is asymptotically stable, a suitable Lyapunov function is constructed explicitly. Choosing in a proper way different operating points, an algorithm is used in Section 5 to stabilize the dynamic system towards the equilibrium point where the maximum production of biogas (methane) is achieved. This problem is known as extremum (peak) seeking. In the literature, extremum seeking algorithms are usually designed in the form of block diagrams (schemes) that are implemented on a bioreactor to tune the dilution rate of the open-loop system, see, e.g., (Marcos et al., 2004; Simeonov et al., 2004; 2007; Wagner et al., 1999). The algorithm presented here, in contrast to the above mentioned, is numerical; it is designed to stabilize the closed-loop system towards the desired maximum operating point. Computer simulations illustrating the robustness of the theoretical results are reported in Section 6. The extremum seeking algorithm is sketched in Appendix.

\section{Model description}

We consider a model of an anaerobic digestion process, based on two main reactions (Bernard et al., 2001; Hess and Bernard, 2008): (a) acidogenesis, where the organic substrate (denoted by $s_{1}$ ) is degraded into volatile fatty acids (VFA, denoted by $s_{2}$ ) by acidogenic bacteria $\left(x_{1}\right)$; (b) methanogenesis, where VFA are degraded into methane $\mathrm{CH}_{4}$ and carbon dioxide $\mathrm{CO}_{2}$ by methanogenic bacteria $\left(x_{2}\right)$. We assume that the methane flow rate is the measurable output and denote it by $Q$. The mass balance model in a continuously stirred tank bioreactor is described by the following nonlinear system of ordinary differential equations:

$$
\begin{aligned}
\frac{\mathrm{d} s_{1}}{\mathrm{~d} t} & =u\left(s_{1}^{i}-s_{1}\right)-k_{1} \cdot \mu_{1}\left(s_{1}\right) \cdot x_{1} \\
\frac{\mathrm{d} x_{1}}{\mathrm{~d} t} & =\left(\mu_{1}\left(s_{1}\right)-\alpha u\right) \cdot x_{1} \\
\frac{\mathrm{d} s_{2}}{\mathrm{~d} t} & =u\left(s_{2}^{i}-s_{2}\right)+k_{2} \cdot \mu_{1}\left(s_{1}\right) \cdot x_{1}-k_{3} \cdot \mu_{2}\left(s_{2}\right) \cdot x_{2}, \\
\frac{\mathrm{d} x_{2}}{\mathrm{~d} t} & =\left(\mu_{2}\left(s_{2}\right)-\alpha u\right) \cdot x_{2}, \\
Q & =k_{4} \cdot \mu_{2}\left(s_{2}\right) \cdot x_{2},
\end{aligned}
$$

where

$$
\begin{aligned}
& \mu_{1}\left(s_{1}\right)=\frac{\mu_{\max } s_{1}}{k_{\mathrm{s}_{1}}+s_{1}}, \\
& \mu_{2}\left(s_{2}\right)=\frac{\mu_{0} s_{2}}{k_{\mathrm{s}_{2}}+s_{2}+\left(\frac{s_{2}}{k_{I}}\right)^{2}}
\end{aligned}
$$

\begin{tabular}{|c|c|}
\hline$s_{1}$ & $\begin{array}{l}\text { concentration of chemical oxygen } \\
\text { demand (COD) }[\mathrm{g} / \mathrm{l}]\end{array}$ \\
\hline$s_{2}$ & $\begin{array}{l}\text { concentration of volatile fatty } \\
\text { acids (VFA) }[\mathrm{mmol} / \mathrm{l}]\end{array}$ \\
\hline$x_{1}$ & concentration of acidogenic bacteria $[\mathrm{g} / \mathrm{l}]$ \\
\hline$x_{2}$ & concentration of methanogenic bacteria $[\mathrm{g} / \mathrm{l}]$ \\
\hline$u$ & dilution rate $\left[\right.$ day $\left.^{-1}\right]$ \\
\hline$s_{1}^{i}$ & influent concentration $s_{1}[\mathrm{~g} / \mathrm{l}]$ \\
\hline$s_{2}^{i}$ & influent concentration $s_{2}[\mathrm{mmol} / \mathrm{l}]$ \\
\hline$k_{1}$ & $\begin{array}{l}\text { yield coefficient for COD } \\
\text { degradation }\left[\mathrm{g} \mathrm{COD} /\left(\mathrm{g} x_{1}\right)\right]\end{array}$ \\
\hline$k_{2}$ & $\begin{array}{l}\text { yield coefficient for VFA } \\
\text { production }\left[\mathrm{mmol} \mathrm{VFA} /\left(\mathrm{g} x_{1}\right)\right]\end{array}$ \\
\hline$k_{3}$ & $\begin{array}{l}\text { yield coefficient for VFA } \\
\text { consumption }\left[\mathrm{mmol} \mathrm{VFA} /\left(\mathrm{g} x_{2}\right)\right]\end{array}$ \\
\hline$k_{4}$ & coefficient $\left[1^{2} / g\right]$ \\
\hline$\mu_{\max }$ & $\begin{array}{l}\text { maximum acidogenic biomass } \\
\text { growth rate }\left[\text { day }^{-1}\right]\end{array}$ \\
\hline$\mu_{0}$ & $\begin{array}{l}\text { maximum methanogenic biomass } \\
\text { growth rate }\left[\text { day }^{-1}\right]\end{array}$ \\
\hline$k_{s_{1}}$ & $\begin{array}{l}\text { saturation parameter associated } \\
\text { with } s_{1}[\mathrm{~g} \text { COD/l] }\end{array}$ \\
\hline$k_{s_{2}}$ & $\begin{array}{l}\text { saturation parameter associated } \\
\text { with } s_{2}[\mathrm{mmol} \mathrm{VFA} / \mathrm{l}]\end{array}$ \\
\hline$k_{I}$ & $\begin{array}{l}\text { inhibition constant associated } \\
\text { with } s_{2}\left[(\mathrm{mmol} \mathrm{VFA} / \mathrm{l})^{1 / 2}\right]\end{array}$ \\
\hline$\alpha$ & $\begin{array}{l}\text { proportion of dilution rate reflecting } \\
\text { process heterogeneity }\end{array}$ \\
\hline$Q$ & methane gas flow rate \\
\hline
\end{tabular}

are model functions of the specific growth rates of the microorganisms. The first one, $\mu_{1}\left(s_{1}\right)$, presents the Monod law; the second one, $\mu_{2}\left(s_{2}\right)$, presents the Haldane law
Table 1. Definition of the model variables and parameters.

and exhibits substrate inhibition, that is, there is a point $\tilde{s}_{2}$ such that $\mu_{2}\left(s_{2}\right)$ achieves its maximum (Bastin and Dochain, 1990),

$$
\tilde{s}_{2}=k_{I} \sqrt{k_{s_{2}}} .
$$

The state variables $s_{1}, s_{2}$ and $x_{1}, x_{2}$ denote substrate and biomass concentrations, respectively: $s_{1}$ represents the organic substrate, characterized by its chemical oxygen demand (COD), $s_{2}$ denotes the volatile fatty acids (VFA), $x_{1}$ and $x_{2}$ are the acidogenic and methanogenic bacteria. The definition of the model parameters is given in Table 1 .

The parameter $\alpha \in[0,1]$ represents the proportion of bacteria that are affected by the dilution; $\alpha=0$ and $\alpha=1$ correspond to an ideal fixed bed reactor and to an ideal continuous stirred tank reactor, respectively (cf., for example, (Alcaraz-González et al., 2002; Antonelli et al., 2003; Bernard et al., 2000; 2001; Grognard and Bernard, 2006; Schoefs et al., 2003)). The dilution rate $u$ is considered as a control variable. 


\section{Bifurcation analysis of the equilibrium points of the open-loop system}

In this section we shall consider the open-loop system (1)(4), that is, we assume that the control input $u$ is a positive parameter.

3.1. Equilibrium points. The equilibrium points are solutions of the nonlinear algebraic system obtained from (1)-(4) by setting the right-hand side functions to zero, that is,

$$
\begin{aligned}
& u \cdot\left(s_{1}^{i}-s_{1}\right)-k_{1} \cdot \mu_{1}\left(s_{1}\right) \cdot x_{1}=0, \\
& \left(\mu_{1}\left(s_{1}\right)-\alpha u\right) \cdot x_{1}=0, \\
& u \cdot\left(s_{2}^{i}-s_{2}\right)+k_{2} \cdot \mu_{1}\left(s_{1}\right) \cdot x_{1}-k_{3} \cdot \mu_{2}\left(s_{2}\right) \cdot x_{2}=0, \\
& \left(\mu_{2}\left(s_{2}\right)-\alpha u\right) \cdot x_{2}=0 .
\end{aligned}
$$

We are looking for nonnegative solutions $s_{j}$ and $x_{j}$, $j=1,2$, of the above system. For that reason, we assume that the following inequalities are valid (Bastin and Dochain, 1990; Hess and Bernard, 2008):

$$
s_{1} \leq s_{1}^{i}, \quad s_{2} \leq s_{2}^{i}+\frac{k_{2}}{k_{1}} s_{1}^{i} .
$$

The second inequality in 111 is motivated by the fact that the imbalance between acidogenesis and methanogenesis might lead to the accumulation of VFA $\left(s_{2}\right)$ and therefore to the acidification $\left(x_{2}=0\right)$ of the bioreactor; thus $s_{2}^{i}+$ $\left(k_{2} / k_{1}\right) s_{1}^{i}$ can be considered as a worst-case upper bound of the total concentration $s_{2}$ (Hess and Bernard, 2008). As mentioned before, the Haldane model function $\mu_{2}\left(s_{2}\right)$ achieves a maximum at the point $\tilde{s}_{2}$ (see (6)). We assume that

$$
\tilde{s}_{2} \leq s_{2}^{i},
$$

otherwise $\mu_{2}\left(s_{2}\right)$ would be monotonically increasing for $s_{2} \geq 0$ as the Monod law $\mu_{1}\left(s_{1}\right)$ for $s_{1} \geq 0$ does.

Equations (7) and (8) are uncoupled with respect to $s_{2}$ and $x_{2}$. They possess a nontrivial solution

$$
\begin{aligned}
& s_{1}^{(1)}(u)=\frac{\alpha u k_{s_{1}}}{\mu_{\max }-\alpha u}, \quad x_{1}^{(1)}(u)=\frac{s_{1}^{i}-s_{1}(u)}{\alpha k_{1}}, \\
& u \in\left(0, \frac{1}{\alpha} \mu_{1}\left(s_{1}^{i}\right)\right)
\end{aligned}
$$

and a trivial solution (called the wash-out state with respect to $x_{1}$ )

$$
s_{1}^{(2)}(u)=s_{1}^{i}, \quad x_{1}^{(2)}(u)=0 \text { for all } u>0 .
$$

Write

$$
u_{1}=\frac{1}{\alpha} \mu_{1}\left(s_{1}^{i}\right) .
$$

Note that $u<u_{1}$ implies $s_{1}^{(1)}(u)<s_{1}^{i}$. For $u=u_{1}$ we get

$$
s_{1}^{(1)}\left(u_{1}\right)=s_{1}^{(2)}\left(u_{1}\right)=s_{1}^{i}, \quad x_{1}^{(1)}\left(u_{1}\right)=x_{1}^{(2)}\left(u_{1}\right)=0 .
$$

Consider (10) and assume first that $x_{2} \neq 0$. Then $\mu_{2}\left(s_{2}\right)-\alpha u=0$ is equivalent to the following quadratic equation with respect to $s_{2}$ :

$$
\frac{\alpha u}{k_{I}^{2}} s_{2}^{2}+\left(\alpha u-\mu_{0}\right) s_{2}+\alpha u k_{s_{2}}=0,
$$

for which the discriminant

$$
\Delta(u)=\alpha^{2}\left(1-4 \frac{k_{s_{2}}}{k_{I}^{2}}\right) u^{2}-2 \alpha \mu_{0} u+\mu_{0}^{2}
$$

vanishes at the points

$$
u_{2}=\frac{\mu_{0}}{\alpha\left(1+2 \sqrt{k_{s_{2}}} / k_{I}\right)}, \quad u^{(2)}=\frac{\mu_{0}}{\alpha\left(1-2 \sqrt{k_{s_{2}}} / k_{I}\right)} .
$$

Obviously, $u_{2}>0$ and $\operatorname{sign} u^{(2)}=\operatorname{sign}\left(1-2 \sqrt{k_{s_{2}}} / k_{I}\right)$.

Let first $u \in\left(0, u_{2}\right]$. Then $\Delta(u) \geq 0$ and $\alpha u<\mu_{0}$ are valid. Thus (15) possesses two positive roots,

$$
\begin{aligned}
& s_{2}^{(1)}(u)=\frac{2 \alpha u k_{s_{2}}}{\mu_{0}-\alpha u+\sqrt{\Delta(u)}}, \\
& s_{2}^{(2)}(u)=\frac{\mu_{0}-\alpha u+\sqrt{\Delta(u)}}{2 \alpha u / k_{I}^{2}} .
\end{aligned}
$$

If $u^{(2)}>0$ is fulfilled, then $\Delta(u) \geq 0$ for $u \in$ $\left[u^{(2)},+\infty\right)$. In this case, $\alpha u>\mu_{0}$ holds true and the two roots (16) and 17) are negative, which is physically impossible. Therefore the admissible interval for the steady states (16), (17) is $\left(0, u_{2}\right]$. It is straightforward to see that

$$
u_{2}=\frac{1}{\alpha} \mu_{2}\left(\tilde{s}_{2}\right)
$$

Further, solving (9) with $s_{1}=s_{1}^{(1)}(u), x_{1}=x_{1}^{(1)}(u)$, we obtain the roots

$$
\begin{aligned}
& x_{2}^{(1)}(u)=\frac{s_{2}^{i}-s_{2}^{(1)}(u)+\alpha k_{2} x_{1}^{(1)}(u)}{\alpha k_{3}}, \\
& x_{2}^{(2)}(u)=\frac{s_{2}^{i}-s_{2}^{(2)}(u)+\alpha k_{2} x_{1}^{(1)}(u)}{\alpha k_{3}} .
\end{aligned}
$$

Substituting $x_{1}^{(1)}(u)$ by $x_{1}^{(2)}(u)=0$ into the above expressions for $x_{2}^{(1)}(u)$ and $x_{2}^{(2)}(u)$, we obtain

$$
x_{2}^{(3)}(u)=\frac{s_{2}^{i}-s_{2}^{(1)}(u)}{\alpha k_{3}}, \quad x_{2}^{(4)}(u)=\frac{s_{2}^{i}-s_{2}^{(2)}(u)}{\alpha k_{3}} .
$$

Assume now that $x_{2}=0$ in (10) holds true, that is, we set

$$
x_{2}^{(5)}=0 \text {. }
$$

Then solving (9) with $s_{1}=s_{1}^{(1)}(u)$ and $x_{1}=x_{1}^{(1)}(u)$ implies

$$
s_{2}^{(3)}(u)=s_{2}^{i}+\frac{k_{2}}{k_{1}}\left(s_{1}^{i}-s_{1}^{(1)}(u)\right) \text { for } u \in\left(0, u_{1}\right) .
$$


Finally, solving (9) with $s_{1}=s_{1}^{i}, x_{1}=0$ leads to

$$
s_{2}^{(4)}(u)=s_{2}^{i} .
$$

All components of the equilibrium points should be nonnegative. This condition determines the admissible values for the parameter $u$. First let us note that $s_{2}^{(1)}(u)$ is a monotonically increasing function of $u$ whereas $s_{2}^{(2)}(u)$ is monotonically decreasing on $\left(0, u_{2}\right]$. Moreover,

$$
\begin{aligned}
& s_{2}^{(1)}(0)=0, \quad s_{2}^{(1)}\left(u_{2}\right)=s_{2}^{(2)}\left(u_{2}\right)=\tilde{s}_{2} \leq s_{2}^{i}, \\
& \lim _{u \rightarrow 0} s_{2}^{(2)}(u)=+\infty .
\end{aligned}
$$

Therefore, $x_{2}^{(3)}(u) \geq 0$ for $u \in\left(0, u_{2}\right]$. Further,

$$
x_{2}^{(4)}(u) \geq 0 \Longleftrightarrow s_{2}^{(2)}(u) \leq s_{2}^{i} \Longleftrightarrow u \leq \frac{1}{\alpha} \mu_{2}\left(s_{2}^{i}\right) \text {. }
$$

Write

$$
u_{3}=\frac{1}{\alpha} \mu_{2}\left(s_{2}^{i}\right) .
$$

According to the assumption $11, s_{2}^{(2)}(u)$ should satisfy

$$
s_{2}^{(2)}(u) \leq s_{2}^{i}+\frac{k_{2}}{k_{1}} s_{1}^{i},
$$

which is possible if and only if

$$
u \geq \frac{1}{\alpha} \mu_{2}\left(s_{2}^{i}+\frac{k_{2}}{k_{1}} s_{1}^{i}\right) .
$$

Write

$$
u_{4}=\frac{1}{\alpha} \mu_{2}\left(s_{2}^{i}+\frac{k_{2}}{k_{1}} s_{1}^{i}\right) .
$$

Further, $x_{2}^{(2)}(u)$ is a monotonically increasing function of $u, \lim _{u \rightarrow 0} x_{2}^{(2)}(u)=-\infty$ and $x_{2}^{(2)}\left(u_{2}\right)>0$. Thus there is a unique point

$$
u_{5} \in\left(0, u_{2}\right) \text { such that } x_{2}^{(2)}\left(u_{5}\right)=0 .
$$

Then $x_{2}^{(2)}(u) \geq 0$ if and only if $u \geq u_{5}$.

Obviously, $\tilde{s}_{2} \leq s_{2}^{i}$ (see (12) ) implies

$$
u_{4}<u_{3} \leq u_{2} \text {. }
$$

Finally, we have the following six equilibrium points

$$
\begin{aligned}
& E_{1}(u)=\left(s_{1}^{(1)}(u), x_{1}^{(1)}(u), s_{2}^{(1)}(u), x_{2}^{(1)}(u)\right), \\
& u \in\left(0, \min \left\{u_{1}, u_{2}\right\}\right],\left(s_{1}^{(1)}(u), x_{1}^{(1)}(u), s_{2}^{(2)}(u), x_{2}^{(2)}(u)\right), \\
& E_{2}(u)=\left[\max \left\{u_{4}, u_{5}\right\}, \min \left\{u_{1}, u_{2}\right\}\right],\left(s_{1}^{(1)}(u), x_{1}^{(1)}(u), s_{2}^{(3)}(u), 0\right), u \in\left(0, u_{1}\right], \\
& E_{3}(u)=\left(0, u_{3}\right], \\
& E_{4}(u)=\left(s_{1}^{i}, 0, s_{2}^{(1)}(u), x_{2}^{(3)}(u)\right), \quad u \in\left[u_{3}, u_{2}\right], \\
& E_{5}(u)=\left(s_{1}^{i}, 0, s_{2}^{(2)}(u), x_{2}^{(4)}(u)\right), \quad u \in \\
& E_{6}(u)=\left(s_{1}^{i}, 0, s_{2}^{i}, 0\right), \quad u \geq 0 .
\end{aligned}
$$

Remark 1. The equilibrium point $E_{2}(u)$ does not exist if $u_{1}<\max \left\{u_{4}, u_{5}\right\}$.

Remark 2. It follows from (9) and (7) that $x_{2}=0$, $s_{2}=s_{2}^{i}$ always leads to $x_{1}=0, s_{1}=s_{1}^{i}$.

Figure 1 presents the curves (branches) of the steady state components as functions of the parameter $u$.

3.2. Local one-parameter bifurcations of the equilibrium points. Denote, for simplicity, $z=$ $\left(s_{1}, x_{1}, s_{2}, x_{2}\right)$ and by $G=G(z ; u)=\left(G_{1}, G_{2}, G_{3}, G_{4}\right)^{T}$ the vector of right-hand side functions of (11)-(4). It is known that if an equilibrium point is hyperbolic, that is, when the linearization (Jacobian matrix) $D G\left(z_{0} ; u_{0}\right)=$ $D_{z} G\left(z_{0} ; u_{0}\right)$ at some equilibrium point $z_{0}$ for some value of $u=u_{0}$ does not possess eigenvalues on the imaginary axis, then $\left(z_{0} ; u_{0}\right)$ is linearly stable or unstable. This means that varying $u$ slightly in a neighborhood of $u_{0}$ will not change the nature of the stability of the steady state (Carr, 1981; Wiggins, 1990). When $\left(z_{0} ; u_{0}\right)$ is not hyperbolic, that is, when $D G\left(z_{0} ; u_{0}\right)$ has some eigenvalues on the imaginary axis, then for $u$ close to $u_{0}$ a new dynamical behavior can occur. In what follows we shall consider the simplest way in which an equilibrium point can be nonhyperbolic, namely, when $D G\left(z_{0} ; u_{0}\right)$ possesses a single zero eigenvalue with the remaining eigenvalues having nonzero real parts.

The Jacobian matrix has the form

$$
D G(z ; u)=\left(\begin{array}{cccc}
\frac{\partial G_{1}}{\partial s_{1}} & \frac{\partial G_{1}}{\partial x_{1}} & 0 & 0 \\
\frac{\partial G_{2}}{\partial s_{1}} & \frac{\partial G_{2}}{\partial x_{1}} & 0 & 0 \\
\frac{\partial G_{3}}{\partial s_{1}} & \frac{\partial G_{3}}{\partial x_{1}} & \frac{\partial G_{3}}{\partial s_{2}} & \frac{\partial G_{3}}{\partial x_{2}} \\
0 & 0 & \frac{\partial G_{4}}{\partial s_{2}} & \frac{\partial G_{4}}{\partial x_{2}}
\end{array}\right) .
$$

Taking into account the particular expressions of the righthand side functions $G_{i}(z ; u), i=1, \ldots, 4$, the determinant $|D G(z ; u)|$ of $D G(z ; u)$ can be formed as a product $|D G(z ; u)|=\left|D^{1} G(z ; u)\right| \cdot\left|D^{2} G(z ; u)\right|$, where

$$
\begin{aligned}
& \left|D^{1} G(z ; u)\right| \\
& \quad=\frac{\partial G_{1}}{\partial s_{1}} \frac{\partial G_{2}}{\partial x_{1}}-\frac{\partial G_{1}}{\partial x_{1}} \frac{\partial G_{2}}{\partial s_{1}} \\
& \quad=-u\left(\mu_{1}\left(s_{1}\right)-\alpha u\right)+\alpha k_{1} u x_{1} \frac{\mathrm{d}}{\mathrm{d} s_{1}} \mu_{1}\left(s_{1}\right), \\
& \left|D^{2} G(z ; u)\right| \\
& \quad=\frac{\partial G_{3}}{\partial s_{2}} \frac{\partial G_{4}}{\partial x_{2}}-\frac{\partial G_{3}}{\partial x_{2}} \frac{\partial G_{4}}{\partial s_{2}} \\
& \quad=u\left(\alpha k_{3} x_{2} \frac{\mathrm{d}}{\mathrm{d} s_{2}} \mu_{2}\left(s_{2}\right)-\left(\mu_{2}\left(s_{2}\right)-\alpha u\right)\right) .
\end{aligned}
$$


(a)

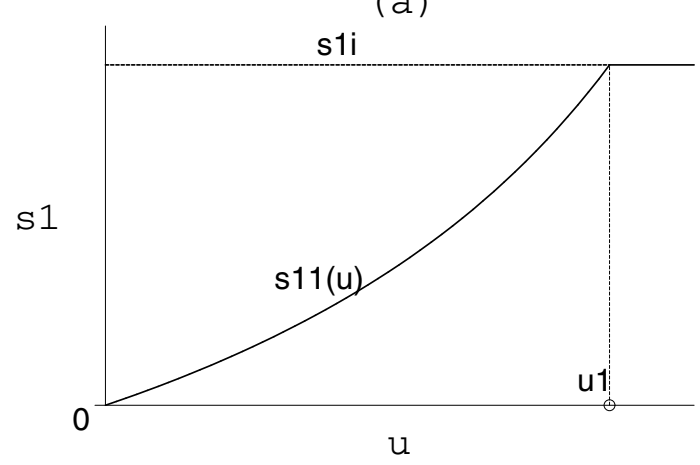

(b)

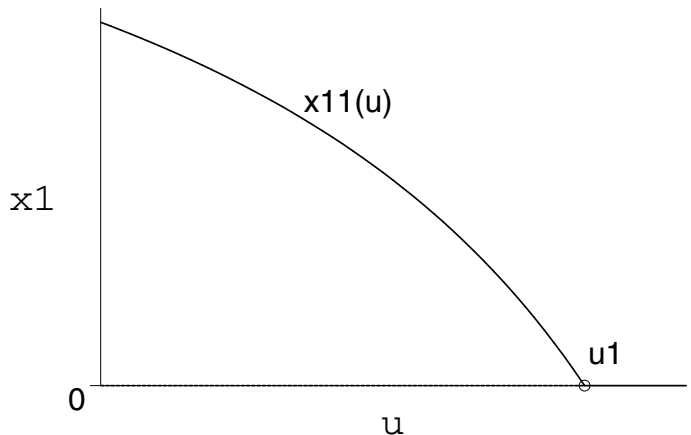

(c)

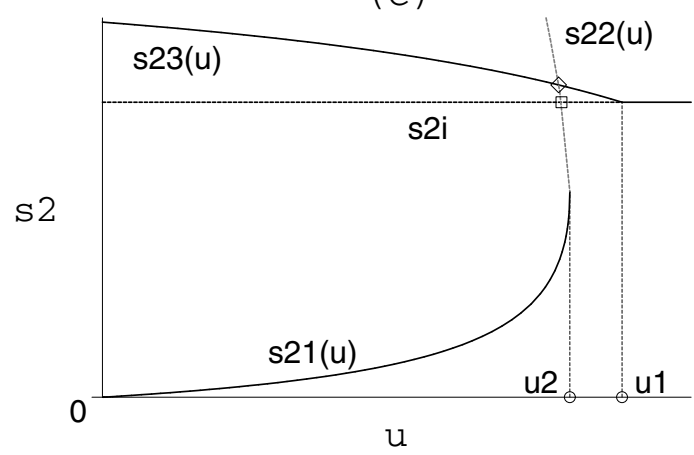

(d)

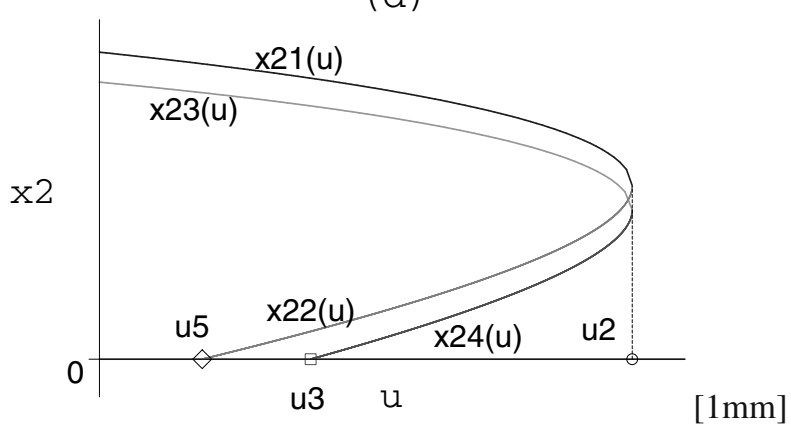

Fig. 1. Branches of equilibrium points vs. $u$ in the case when $u_{2}<u_{1}$ : (a) $s_{1}$-components, (b) $x_{1}$-components, (c) $s_{2}$-components, (d) $x_{2}$-components. Notation: (a) s1i $:=s_{1}^{i}, \mathrm{~s} 11(\mathrm{u}):=s_{1}^{(1)}(u) ;(\mathrm{b}) \mathrm{x} 11(\mathrm{u}):=x_{1}^{(1)}(u)$; (c) $\mathrm{s} 2 \mathrm{i}:=s_{2}^{i}$, $\mathrm{s} 2 \mathrm{j}(\mathrm{u}):=s_{2}^{(j)}(u), j=1,2,3$; (d) $\mathrm{x} 2 \mathrm{j}(\mathrm{u}):=x_{2}^{(j)}(u), j=$ $1,2,3,4$.
In what follows we shall assume that $\left|D^{1} G(z ; u)\right|$ and $\left|D^{2} G(z ; u)\right|$ do not vanish simultaneously at an equilibrium point.

Case 1. $\left|D^{1} G(z ; u)\right|=0,\left|D^{2} G(z ; u)\right| \neq 0$.

In this case we have the following nonhyperbolic equilibrium points:

$$
\begin{aligned}
H_{1}= & \left(s_{1}^{i}, 0, s_{2}^{(1)}\left(u_{1}\right), \frac{s_{2}^{i}-s_{2}^{(1)}\left(u_{1}\right)}{\alpha k_{3}} ; u_{1}\right) \\
& \text { if } u_{1}<u_{3} \leq u_{2}, \\
H_{2}= & \left(s_{1}^{i}, 0, s_{2}^{(2)}\left(u_{1}\right), \frac{s_{2}^{i}-s_{2}^{(2)}\left(u_{1}\right)}{\alpha k_{3}} ; u_{1}\right) \\
& \text { if } u_{3}<u_{1}<u_{2}, \\
H_{3}= & \left(s_{1}^{i}, 0, s_{2}^{i}, 0 ; u_{1}\right) \text { if } u_{1}<u_{2}, u_{1} \neq u_{3} .
\end{aligned}
$$

Case 2. $\left|D^{1} G(z ; u)\right| \neq 0,\left|D^{2} G(z ; u)\right|=0$.

The nonhyperbolic equilibrium points are

$$
\begin{aligned}
H_{4}= & \left(s_{1}^{i}, 0, s_{2}^{i}, 0 ; u_{3}\right) \text { if } u_{1}<u_{3}, \\
H_{5}= & \left(s_{1}^{i}, 0, \tilde{s}_{2}, \frac{s_{2}^{i}-\tilde{s}_{2}}{\alpha k_{3}} ; u_{2}\right) \text { if } u_{1}<u_{2}, u_{2} \neq u_{3}, \\
H_{6}= & \left(s_{1}^{(1)}\left(u_{2}\right), x_{1}^{(1)}\left(u_{2}\right), \tilde{s}_{2},\right. \\
& \left.\frac{s_{2}^{i}-\tilde{s}_{2}+\alpha k_{2} x_{1}^{(1)}\left(u_{2}\right)}{\alpha k_{3}} ; u_{2}\right) \text { if } u_{2}<u_{1}, \\
H_{7}= & \left(s_{1}^{(1)}\left(u_{2}\right), x_{1}^{(1)}\left(u_{2}\right), s_{2}^{i}, \frac{k_{2}}{k_{3}} x_{1}^{(1)}\left(u_{2}\right) ; u_{2}\right) \\
& \text { if } u_{3}=u_{2}<u_{1}, \\
H_{8}= & \left(s_{1}^{(1)}\left(u_{5}\right), x_{1}^{(1)}\left(u_{5}\right), s_{2}^{(3)}\left(u_{5}\right), 0 ; u_{5}\right) \text { if } u_{5}<u_{1} .
\end{aligned}
$$

Our goal is to determine the nature of the stability of $H_{j}, j=1, \ldots, 8$, for $u$ near the corresponding critical value $u_{i}$. To do this, we shall find the reduction of the system (1)-(4) at each nonhyperbolic point to its corresponding center manifold (Carr, 1981; Wiggins, 1990).

Proposition 1. The system of ODEs (17)-(4) undergoes a transcritical bifurcation at each one of the nonhyperbolic points $H_{j}, j=1,2, \ldots, 8$.

Proof. We shall consider in detail the point $H_{8}$. Write, for simplicity,

$$
s_{1}^{*}=s_{1}^{(1)}\left(u_{5}\right), \quad x_{1}^{*}=x_{1}^{(1)}\left(u_{5}\right), \quad s_{2}^{*}=s_{2}^{(3)}\left(u_{5}\right) .
$$

The following coordinate change

$$
\begin{array}{lll}
\xi_{1}=s_{1}^{*}-s_{1}, & \eta_{1}=x_{1}^{*}-x_{1}, & \xi_{2}=s_{2}^{*}-s_{2}, \\
\eta_{2}=x_{2}, & v=u_{5}-u
\end{array}
$$

transforms $H_{8}$ into zero $(0,0,0,0 ; 0)=:(0 ; 0)$. Using Taylor approximations of $\mu_{1}\left(s_{1}^{*}-\xi_{1}\right)$ and $\mu_{2}\left(s_{2}^{*}-\xi_{2}\right)$ 
Table 2.

\begin{tabular}{|c|c|c|}
\hline Nonhyperbolic points & Eigenvalues & Values of $L$ \\
\hline $\begin{array}{c}H_{1}= \\
\left(s_{1}^{i}, 0, s_{2}^{(1)}\left(u_{1}\right), x_{2}^{*} ; u_{1}\right) \\
u_{1}<u_{3} \leq u_{2} \\
x_{2}^{*}:=\frac{s_{2}^{i}-s_{2}^{(1)}\left(u_{1}\right)}{\alpha k_{3}}\end{array}$ & $\begin{array}{l}\lambda_{1}=0, \lambda_{2}=-u_{1} \\
\lambda_{3,4}=\frac{1}{2}\left(u_{1}+k_{3} m_{2} x_{2}^{*}\right) \\
\pm \frac{1}{2} \sqrt{\left(u_{1}+k_{3} m_{2} x_{2}^{*}\right)^{2}-4 \alpha u_{1} k_{3} m_{2} x_{2}^{*}} \\
m_{2}:=\frac{\mathrm{d}}{\mathrm{d} s_{2}} \mu_{2}\left(s_{2}^{(1)}\left(u_{1}\right)\right)\end{array}$ & $\frac{k_{1}}{k_{2}} \alpha k_{3} \frac{\mathrm{d}}{\mathrm{d} s_{1}} \mu_{1}\left(s_{1}^{i}\right)$ \\
\hline $\begin{array}{c}H_{2}= \\
\left(s_{1}^{i}, 0, s_{2}^{(2)}\left(u_{1}\right), x_{2}^{*} ; u_{1}\right) \\
u_{3}<u_{1}<u_{2} \\
x_{2}^{*}:=\frac{s_{2}^{i}-s_{2}^{(2)}\left(u_{1}\right)}{\alpha k_{3}}\end{array}$ & $\begin{array}{l}\lambda_{1}=0, \lambda_{2}=-u_{1} \\
\lambda_{3,4}=\frac{1}{2}\left(u_{1}+k_{3} m_{2} x_{2}^{*}\right) \\
\pm \frac{1}{2} \sqrt{\left(u_{1}+k_{3} m_{2} x_{2}^{*}\right)^{2}-4 \alpha u_{1} k_{3} m_{2} x_{2}^{*}} \\
m_{2}:=\frac{\mathrm{d}}{\mathrm{d} s_{2}} \mu_{2}\left(s_{2}^{(2)}\left(u_{1}\right)\right)\end{array}$ & $\frac{k_{1}}{k_{2}} \alpha k_{3} \frac{\mathrm{d}}{\mathrm{d} s_{1}} \mu_{1}\left(s_{1}^{i}\right)$ \\
\hline $\begin{array}{c}H_{3}=\left(s_{1}^{i}, 0, s_{2}^{i}, 0 ; u_{1}\right) \\
u_{1}<u_{2}, \quad u_{1} \neq u_{3}\end{array}$ & $\lambda_{1}=0, \lambda_{2}=\lambda_{3}=-u_{1}, \lambda_{4}=\alpha\left(u_{3}-u_{1}\right)$ & $\alpha k_{1} \frac{\mathrm{d}}{\mathrm{d} s_{1}} \mu_{1}\left(s_{1}^{i}\right)$ \\
\hline $\begin{aligned} H_{4}= & \left(s_{1}^{i}, 0, s_{2}^{i}, 0 ; u_{3}\right) \\
& u_{1}<u_{3}\end{aligned}$ & $\lambda_{1}=0, \lambda_{2}=\lambda_{3}=-u_{3}, \lambda_{4}=\alpha\left(u_{1}-u_{3}\right)$ & $\alpha k_{3} \frac{\mathrm{d}}{\mathrm{d} s_{2}} \mu_{2}\left(s_{2}^{i}\right)$ \\
\hline $\begin{array}{c}H_{5}=\left(s_{1}^{i}, 0, \tilde{s}_{2}, \frac{s_{2}^{2}-\tilde{s}_{2}}{\alpha k_{3}} ; u_{2}\right) \\
u_{1}<u_{2}, \quad u_{2} \neq u_{3}\end{array}$ & $\lambda_{1}=0, \lambda_{2}=\lambda_{3}=-u_{2}, \lambda_{4}=\alpha\left(u_{1}-u_{2}\right)$ & $\frac{1}{2}\left(s_{2}^{i}-\tilde{s}_{2}\right) \frac{\mathrm{d}^{2}}{\mathrm{~d} s_{2}^{2}} \mu_{2}\left(\tilde{s}_{2}\right)$ \\
\hline $\begin{array}{c}H_{6}= \\
\left(s_{1}^{(1)}\left(u_{2}\right), x_{1}^{(1)}\left(u_{2}\right), \tilde{s}_{2}, x_{2}^{*} ; u_{2}\right) \\
u_{2}<u_{1} \\
x_{2}^{*}:=\frac{s_{2}^{i}-\tilde{s}_{2}+\alpha k_{2} x_{1}^{(1)}\left(u_{2}\right)}{\alpha k_{3}}\end{array}$ & $\begin{array}{l}\lambda_{1}=0, \lambda_{2}=-u_{2} \\
\lambda_{3,4}=-\frac{1}{2}\left(u_{2}+k_{1} m_{1} x_{1}^{(1)}\left(u_{2}\right)\right) \\
\pm \frac{1}{2} \sqrt{\left(u_{2}+k_{1} m_{1} x_{1}^{(1)}\left(u_{2}\right)\right)^{2}-4 \alpha u_{2} k_{1} m_{1} x_{1}^{(1)}\left(u_{2}\right)} \\
m_{1}:=\frac{\mathrm{d}}{\mathrm{d} s_{1}} \mu_{1}\left(s_{1}^{(1)}\left(u_{2}\right)\right)\end{array}$ & $\frac{1}{2} \alpha^{2} k_{3}^{2} \frac{\mathrm{d}^{2}}{\mathrm{~d} s_{2}^{2}} \mu_{2}\left(\tilde{s}_{2}\right) x_{2}^{*}$ \\
\hline $\begin{array}{c}H_{7}= \\
\left(s_{1}^{(1)}\left(u_{2}\right), x_{1}^{(1)}\left(u_{2}\right), s_{2}^{i}, x_{2}^{*} ; u_{2}\right) \\
u_{3}=u_{2}<u_{1} \\
x_{2}^{*}:=\frac{k_{2}}{k_{3}} x_{1}^{(1)}\left(u_{2}\right)\end{array}$ & $\begin{array}{l}\lambda_{1}=0, \lambda_{2}=-u_{2} \\
\lambda_{3,4}=-\frac{1}{2}\left(u_{2}+k_{1} m_{1} x_{1}^{(1)}\left(u_{2}\right)\right) \\
\pm \frac{1}{2} \sqrt{\left(u_{2}+k_{1} m_{1} x_{1}^{(1)}\left(u_{2}\right)\right)^{2}-4 \alpha u_{2} k_{1} m_{1} x_{1}^{(1)}\left(u_{2}\right)} \\
m_{1}:=\frac{\mathrm{d}}{\mathrm{d} s_{1}} \mu_{1}\left(s_{1}^{(1)}\left(u_{2}\right)\right)\end{array}$ & $\frac{1}{2} \alpha^{2} k_{3}^{2} \frac{\mathrm{d}^{2}}{\mathrm{~d} s_{2}^{2}} \mu_{2}\left(\tilde{s}_{2}\right) x_{2}^{*}$ \\
\hline $\begin{array}{c}H_{8}= \\
\left(s_{1}^{(1)}\left(u_{5}\right), x_{1}^{(1)}\left(u_{5}\right), s_{2}^{(3)}\left(u_{5}\right), 0 ; u_{5}\right) \\
u_{5}<u_{1}\end{array}$ & $\begin{array}{l}\lambda_{1}=0, \lambda_{2}=-u_{5} \\
\lambda_{3,4}=-\frac{1}{2}\left(u_{2}+k_{1} m_{1} x_{1}^{(1)}\left(u_{5}\right)\right) \\
\pm \frac{1}{2} \sqrt{\left(u_{5}+k_{1} m_{1} x_{1}^{(1)}\left(u_{5}\right)\right)^{2}-4 \alpha u_{5} k_{1} m_{1} x_{1}^{(1)}\left(u_{5}\right)} \\
m_{1}:=\frac{\mathrm{d}}{\mathrm{d} s_{1}} \mu_{1}\left(s_{1}^{(1)}\left(u_{5}\right)\right)\end{array}$ & $\alpha k_{3} \frac{\mathrm{d}^{2}}{\mathrm{~d} s_{2}^{2}} \mu_{2}\left(s_{2}^{(3)}\left(u_{5}\right)\right)$ \\
\hline
\end{tabular}

around $\xi_{1}=0$ and $\xi_{2}=0$, we obtain

$$
\begin{aligned}
& \mu_{1}\left(s_{1}^{*}-\xi_{1}\right)=\mu_{1}\left(s_{1}^{*}\right)-\frac{\mathrm{d} \mu_{1}}{\mathrm{~d} s_{1}}\left(s_{1}^{*}\right) \cdot \xi_{1}=\alpha u_{5}-m_{1} \xi_{1}, \\
& \mu_{2}\left(s_{2}^{*}-\xi_{2}\right)=\mu_{2}\left(s_{1}^{*}\right)-\frac{\mathrm{d} \mu_{2}}{\mathrm{~d} s_{2}}\left(s_{2}^{*}\right) \cdot \xi_{2}=\alpha u_{5}-m_{2} \xi_{2},
\end{aligned}
$$

where

$$
m_{1}:=\frac{\mathrm{d} \mu_{1}}{\mathrm{~d} s_{1}}\left(s_{1}^{*}\right), \quad m_{2}:=\frac{\mathrm{d} \mu_{2}}{\mathrm{~d} s_{2}}\left(s_{2}^{*}\right) .
$$

Denote by $\widetilde{G}$ the vector of the right hand-side functions of the system (1)-(4) in the new coordinates $\left(\xi_{1}, \eta_{1}, \xi_{2}, \eta_{2}\right)^{T}$. The latter is then expressed in the following form, where the parameter $v$ has been included as a formal dependent variable:

$$
\begin{gathered}
\left(\begin{array}{c}
\dot{\xi}_{1} \\
\dot{\eta}_{1} \\
\dot{\xi}_{2} \\
\dot{\eta}_{2}
\end{array}\right)=D \widetilde{G}(0 ; 0) \cdot\left(\begin{array}{c}
\xi_{1} \\
\eta_{1} \\
\xi_{2} \\
\eta_{2}
\end{array}\right)+\left(\begin{array}{c}
g_{1} \\
g_{2} \\
g_{3} \\
g_{4}
\end{array}\right), \\
\dot{v}=0
\end{gathered}
$$

within

$$
D \widetilde{G}(0 ; 0)
$$

$$
=\left(\begin{array}{cccc}
-\left(u_{5}+k_{1} m_{1} x_{1}^{*}\right) & -\alpha k_{1} u_{5} & 0 & 0 \\
m_{1} x_{1}^{*} & 0 & 0 & 0 \\
k_{2} m_{1} x_{1}^{*} & \alpha k_{2} u_{5} & -u_{5} & \alpha k_{3} u_{5} \\
0 & 0 & 0 & 0
\end{array}\right)
$$


and

$$
\begin{aligned}
& g_{1}=v \xi_{1}+k_{1} m_{1} \xi_{1} \eta_{1}, \\
& g_{2}=\alpha v \eta_{1}-m_{1} \xi_{1} \eta_{1}, \\
& g_{3}=v \xi_{2}-k_{1} m_{1} \xi_{1} \eta_{1}-k_{3} m_{2} \xi_{2} \eta_{2}, \\
& g_{4}=\alpha v \eta_{2}-m_{2} \xi_{2} \eta_{2} .
\end{aligned}
$$

The eigenvalues $\lambda_{i}$ and the corresponding eigenvectors $p_{i}$, $i=1,2,3,4$, of $D \tilde{G}(0 ; 0)$ are respectively

$$
\begin{aligned}
\lambda_{1}= & 0, \quad p_{1}=\left(0,0, \alpha k_{3}, 1\right)^{T}, \\
\lambda_{2}= & -u_{5}, \quad p_{2}=(0,0,1,0)^{T}, \\
\lambda_{3}= & -\frac{1}{2}\left(u_{5}+k_{1} m_{1} x_{1}^{*}\right) \\
& +\frac{1}{2} \sqrt{\left(u_{5}+k_{1} m_{1} x_{1}^{*}\right)^{2}-4 \alpha k_{1} m_{1} u_{5} x_{1}^{*}}, \\
p_{3}= & \left(-\frac{k_{1}}{k_{2}},-\frac{\lambda_{4}}{\alpha k_{2} u_{5}}, 1,0\right)^{T}, \\
\lambda_{4}= & -\frac{1}{2}\left(u_{5}+k_{1} m_{1} x_{1}^{*}\right) \\
& -\frac{1}{2} \sqrt{\left(u_{5}+k_{1} m_{1} x_{1}^{*}\right)^{2}-4 \alpha k_{1} m_{1} u_{5} x_{1}^{*}}, \\
p_{4}= & \left(-\frac{k_{1}}{k_{2}},-\frac{\lambda_{3}}{\alpha k_{2} u_{5}}, 1,0\right)^{T} .
\end{aligned}
$$

Obviously, $\lambda_{2}<0$. Since $0<\alpha<1$, it can be easily seen that $\lambda_{3}$ and $\lambda_{4}$ are real negative numbers.

Forming the matrix $P$ by taking as columns the eigenvectors $p_{j}, j=1,2,3,4$,

$$
P=\left(\begin{array}{llll}
p_{1} & p_{2} & p_{3} & p_{4}
\end{array}\right),
$$

and finding its inverse $P^{-1}$, we make the coordinate change

$$
\left(\begin{array}{l}
\xi_{1} \\
\eta_{1} \\
\xi_{2} \\
\eta_{2}
\end{array}\right)=P\left(\begin{array}{l}
\zeta_{1} \\
\varkappa_{1} \\
\zeta_{2} \\
\varkappa_{2}
\end{array}\right), \quad\left(\begin{array}{l}
\zeta_{1} \\
\varkappa_{1} \\
\zeta_{2} \\
\varkappa_{2}
\end{array}\right)=P^{-1}\left(\begin{array}{l}
\xi_{1} \\
\eta_{1} \\
\xi_{2} \\
\eta_{2}
\end{array}\right)
$$

and obtain

$$
\underset{\dot{v}=0,}{\left(\begin{array}{l}
\dot{\zeta}_{1} \\
\dot{\varkappa}_{1} \\
\dot{\zeta}_{2} \\
\dot{\varkappa}_{2}
\end{array}\right)}=A \cdot\left(\begin{array}{c}
\zeta_{1} \\
\varkappa_{1} \\
\zeta_{2} \\
\varkappa_{2}
\end{array}\right)+\left(\begin{array}{c}
f_{1} \\
f_{2} \\
f_{3} \\
f_{4}
\end{array}\right)
$$

where

$$
A=\left(\begin{array}{cccc}
0 & 0 & 0 & 0 \\
0 & \lambda_{2} & 0 & 0 \\
0 & 0 & \lambda_{3} & 0 \\
0 & 0 & 0 & \lambda_{4}
\end{array}\right)
$$

and

$$
\begin{aligned}
f_{1}= & \alpha v \zeta_{1}-m_{2} \zeta_{1}\left(\alpha k_{3} \zeta_{1}+\varkappa_{1}+\zeta_{2} \varkappa_{2}\right), \\
f_{2}= & v\left(\zeta_{2}+\varkappa_{2}\right)-\alpha^{2} k_{2} v \zeta_{1} \\
& +(\alpha-1) k_{3} m_{2} \zeta_{1}\left(\alpha k_{3} \zeta_{1}+\varkappa_{1}+\zeta_{2}+\varkappa_{2}\right), \\
f_{3}= & \frac{1}{\lambda_{4}-\lambda_{3}}\left[-\lambda_{3} v\left(\zeta_{2}+\varkappa_{2}\right)+\alpha v\left(\lambda_{4} \zeta_{2}+\lambda_{3} \varkappa_{2}\right)\right. \\
& +\frac{k_{1}}{k_{2}} m_{1}\left(\zeta_{2}+\varkappa_{2}\right)\left(\lambda_{4} \zeta_{2}+\lambda_{3} \varkappa_{2}\right) \\
& \left.+\frac{k_{1} m_{1} \lambda_{3}}{\alpha k_{2} u_{5}}\left(\zeta_{2}+\varkappa_{2}\right)\left(\lambda_{4} \zeta_{2}+\lambda_{3} \varkappa_{2}\right)\right], \\
f_{4}= & \frac{1}{\lambda_{4}-\lambda_{3}}\left[\lambda_{4} v\left(\zeta_{2}+\varkappa_{2}\right)-\alpha v\left(\lambda_{4} \zeta_{2}+\lambda_{3} \varkappa_{2}\right)\right. \\
& -\frac{k_{1}}{k_{2}} m_{1}\left(\zeta_{2}+\varkappa_{2}\right)\left(\lambda_{4} \zeta_{2}+\lambda_{3} \varkappa_{2}\right) \\
& \left.-\frac{k_{1} m_{1} \lambda_{4}}{\alpha k_{2} u_{5}}\left(\zeta_{2}+\varkappa_{2}\right)\left(\lambda_{4} \zeta_{2}+\lambda_{3} \varkappa_{2}\right)\right] .
\end{aligned}
$$

Thus, from center manifold theory, the stability of $\left(\xi_{1}, \eta_{1}, \xi_{2}, \eta_{2}\right)=(0,0,0,0)$ near $v=0$ can be determined by studying a one-parameter family of first-order ODEs on a center manifold. The latter can be represented as a graph over $\zeta_{1}$ and $v$ by

$W^{c}(0)$

$$
\begin{aligned}
& =\left\{\left(\zeta_{1}, \varkappa_{1}, \zeta_{2}, \varkappa_{2} ; v\right) \in \mathbb{R}^{5} \mid \varkappa_{1}=h_{1}\left(\zeta_{1}, v\right),\right. \\
& \zeta_{2}=h_{2}\left(\zeta_{1}, v\right), \varkappa_{2}=h_{3}\left(\zeta_{1}, v\right), h_{i}(0,0)=0, \\
& \left.D_{\zeta_{1}} h_{i}(0,0)=0, i=1,2,3\right\}
\end{aligned}
$$

for $\zeta_{1}$ and $v$ sufficiently small. The points $\left(h_{1}, h_{2}, h_{3}\right)^{T}$ of the center manifold should satisfy

$$
D_{\zeta_{1}}\left(\begin{array}{c}
h_{1} \\
h_{2} \\
h_{3}
\end{array}\right) \cdot f_{1}-B \cdot\left(\begin{array}{c}
h_{1} \\
h_{2} \\
h_{3}
\end{array}\right)-\left(\begin{array}{c}
f_{2} \\
f_{3} \\
f_{4}
\end{array}\right)=0
$$

within

$$
B=\left(\begin{array}{ccc}
-u_{5} & 0 & 0 \\
0 & \lambda_{3} & 0 \\
0 & 0 & \lambda_{4}
\end{array}\right) .
$$

We now wish to compute the center manifold $W^{c}(0)$ approximately and to derive the dynamics on $W^{c}(0)$. We assume that

$$
\begin{aligned}
& h_{1}=h_{1}\left(\zeta_{1}, v\right)=a_{1} \zeta_{1}^{2}+a_{2} \zeta_{1} v+a_{3} v^{2}+O(3), \\
& h_{2}=h_{2}\left(\zeta_{1}, v\right)=b_{1} \zeta_{1}^{2}+b_{2} \zeta_{1} v+b_{3} v^{2}+O(3), \\
& h_{3}=h_{3}\left(\zeta_{1}, v\right)=c_{1} \zeta_{1}^{2}+c_{2} \zeta_{1} v+c_{3} v^{2}+O(3) .
\end{aligned}
$$

Substituting this in (20) and equating terms with equal powers to zero, we find

$$
\begin{aligned}
& h_{1}\left(\zeta_{1}, v\right)=\frac{\alpha(\alpha-1) k_{3}^{2} m_{2}}{u_{5}} \zeta_{1}^{2}+\frac{\alpha^{2} k_{2}}{u_{5}} \zeta_{1} v+O(3), \\
& b_{1}=b_{2}=b_{3}=0, \quad c_{1}=c_{2}=c_{3}=0 .
\end{aligned}
$$


Hence the system of ODEs reduced to the center manifold is given by

$$
\begin{aligned}
\dot{\zeta}_{1} & =\alpha v \zeta_{1}-\alpha k_{3} m_{2} \zeta_{1}^{2}, \\
\dot{v} & =0 .
\end{aligned}
$$

Obviously, $\zeta_{1}=0$ is always an equilibrium point for the first equation of (21) and it is stable for $v<0$ and unstable for $v>0$. A change in stability occurs at the bifurcation value $v=0$; the type of bifurcation of $\zeta_{1}=0$ is transcritical (Wiggins, 1990). Since the remaining eigenvalues $\lambda_{2}, \lambda_{3}$ and $\lambda_{4}$ are negative, it follows (in $(z ; u)$ coordinates) that $H_{8}$ is a stable equilibrium for the system (1)-(4) if $u$ is sufficiently close to $u_{5}$ with $u_{5}<u<u_{1}$.

In a similar way it can be shown that the system of ODEs (1)-(4) undergoes a transcritical bifurcation at $H_{j}$, $j=1,2, \ldots, 7$. In all cases the reduced dynamics on the center manifold are of the form

$$
\begin{aligned}
\dot{\zeta}_{1} & =\alpha v \zeta_{1}-L \zeta_{1}^{2}, \\
\dot{v} & =0 .
\end{aligned}
$$

Table 2 presents the eigenvalues and the constant $L$ for all nonhyperbolic points $H_{i}, i=1, \ldots, 8\left(H_{8}\right.$ is included for convenience). It is easy to see that $H_{3}$ can have one positive eigenvalue $\lambda_{4}$ if $u_{3}>u_{1}$. The remaining nonhyperbolic points are stable for $u$ sufficiently close to and greater than the corresponding bifurcation value.

At transcritical bifuracion points, two steady states coalesce and exchange stability at the bifurcation value. In the $(z ; u)$-coordinates the following pairs of equilibrium points coalesce at the bifurcation points (see Fig. 1):

$$
\begin{array}{ll}
E_{1}\left(u_{1}\right) \equiv E_{4}\left(u_{1}\right) \equiv H_{1}, & E_{2}\left(u_{1}\right) \equiv E_{5}\left(u_{1}\right) \equiv H_{2}, \\
E_{3}\left(u_{1}\right) \equiv E_{6}\left(u_{1}\right) \equiv H_{3}, & E_{5}\left(u_{3}\right) \equiv E_{6}\left(u_{3}\right) \equiv H_{4}, \\
E_{4}\left(u_{2}\right) \equiv E_{5}\left(u_{2}\right) \equiv H_{5}, & E_{1}\left(u_{2}\right) \equiv E_{2}\left(u_{2}\right) \equiv H_{6}, \\
E_{1}\left(u_{3}\right) \equiv E_{2}\left(u_{3}\right) \equiv H_{7}, & E_{2}\left(u_{5}\right) \equiv E_{3}\left(u_{5}\right) \equiv H_{8} .
\end{array}
$$

Remark 3. If we consider (7)-(8), it is easily seen that $s_{1}^{(1)}(u), x_{1}^{(1)}(u)$ are linearly stable (attractors, with two negative eigenvalues of the Jacobian) for any value of $u$ with $u<u_{1}$. The second (wash-out) equilibrium $s_{1}^{(2)}(u)=s_{1}^{i}, x_{1}^{(2)}(u)=0$ is a saddle for $u<u_{1}$ and it is linearly stable if $u>u_{1}$. It is shown in (Hess and Bernard, 2008) that $\left(s_{1}^{(1)}(u), x_{1}^{(1)}(u)\right)$ is globally asymptotically stable for any $u<u_{1}$.

Remark 4. The values of $u_{1}=(1 / \alpha) \mu_{1}\left(s_{1}^{i}\right)$ and $u_{2}=(1 / \alpha) \mu_{2}\left(\tilde{s}_{2}\right)$ are uncoupled; they depend on the particular coefficient values in the expressions of $\mu_{1}$ and $\mu_{2}$. If $u_{2}<u_{1}$, then all bifurcation values $u_{i}$ will satisfy $u_{i}<u_{1}$ (see (18), (19)), i.e., the bifurcations will be related to the $s_{2}$ - and $x_{2}$-components of the steady states. Thus $\left(s_{1}^{(1)}(u), x_{1}^{(1)}(u)\right)$ will be a stable equilibrium point for the acidogenic stage (described by (1)-(2)) whereas the methanogenic phase (Eqns. (3)-(4)) might undergo transcritical bifurcations.

\section{Adaptive asymptotic stabilization}

As mentioned before, the model (1)-(4) describes a twostage process where the first two ODEs, (1)-(2), present the acidogenic phase and the second two, (3)-(4), the methanogenic phase. In practice, acidogenesis is much faster than the methanogenic phase. Moreover the latter phase can be inhibited, which on the one hand is limiting and on the other determining for the whole process (Hess and Bernard, 2008).

In what follows we shall assume that the first stage of the process is already stabilized to some previously chosen point $\left(s_{1}^{*}, x_{1}^{*}\right)$ such that $0<s_{1}^{*}<s_{1}^{i}, x_{1}^{*}>0$ (Simeonov et al., 2004; Simeonov et al., 2007). Write

$$
c_{1}^{*}=\frac{k_{2}}{k_{1}}\left(s_{1}^{i}-s_{1}^{*}\right)
$$

In (Antonelli et al., 2003), where a similar approach to asymptotic stabilization of the model is used, the constant $c_{1}^{*}$ is assumed to be negligible and thus set to 0 . We assume here that $c_{1}^{*}>0$. Then the methanogenic phase of the system is described by the following ODEs:

$$
\begin{aligned}
\frac{\mathrm{d} s_{2}}{\mathrm{~d} t} & =u\left(s_{2}^{i}-s_{2}\right)+c_{1}^{*} u-k_{3} \cdot \mu_{2}\left(s_{2}\right) \cdot x_{2}, \\
\frac{\mathrm{d} x_{2}}{\mathrm{~d} t} & =\left(\mu_{2}\left(s_{2}\right)-\alpha u\right) \cdot x_{2} .
\end{aligned}
$$

Practically, the output methane flow rate $Q$ (see (5) and the substrate concentration $s_{2}^{i}, s_{2}$ are the only measurable variables. Our goal is to construct a smooth feedback law for asymptotic stabilization of 22 - 23) to some previously chosen operating (reference) point $\bar{s}_{2}$. Let us fix such a point $\bar{s}_{2} \in\left(0, s_{2}^{i}\right)$. Then $\left(\bar{s}_{2}, \bar{x}_{2}\right)$ with

$$
\bar{x}_{2}=\frac{s_{2}^{i}+c_{1}^{*}-\bar{s}_{2}}{\alpha k_{3}}
$$

is an equilibrium point for (22)-23).

Our main assumption is that $\alpha, k_{3}, k_{4}, \mu_{0}, k_{s_{2}}$ and $k_{I}$ are unknown but bounded within compact intervals $[\alpha]$, $\left[k_{3}\right],\left[k_{4}\right],\left[\mu_{0}\right],\left[k_{s_{2}}\right]$ and $\left[k_{I}\right]$, respectively.

Let $\omega=\left(\alpha, k_{3}, k_{4}, \mu_{0}, k_{s_{2}}, k_{I}\right)$ be the vector of the exact (unknown) values of the model parameters. Set

$$
\bar{\beta}=\frac{k_{3}}{k_{4}\left(s_{2}^{i}+c_{1}^{*}-\bar{s}_{2}\right)},
$$

and let $\beta^{-}>0$ and $\beta^{+}>0$ be arbitrary real numbers such that $\bar{\beta} \in\left(\beta^{-}, \beta^{+}\right)$.

Following (Antonelli et al., 2003), we extend the system (22) -23 by adding the differential equation

$$
\frac{\mathrm{d} \beta}{\mathrm{d} t}=-C\left(\beta-\beta^{-}\right)\left(\beta^{+}-\beta\right) k_{4} \mu_{2}\left(s_{2}\right) x_{2}\left(s_{2}-\bar{s}_{2}\right),
$$

where $C>0$ is an arbitrary constant. 
Define the following map:

$$
\begin{aligned}
& K\left(s_{2}, x_{2}, \beta\right) \\
& =\left\{\begin{array}{c}
\beta k_{4} \mu_{2}\left(s_{2}\right) x_{2}-\gamma\left(s_{2}-\bar{s}_{2}\right) \\
\quad \text { if } \beta k_{4} \mu_{2}\left(s_{2}\right) x_{2}-\gamma\left(s_{2}-\bar{s}_{2}\right)>0 \\
\beta k_{4} \mu_{2}\left(s_{2}\right) x_{2} \\
\quad \text { if } \beta k_{4} \mu_{2}\left(s_{2}\right) x_{2}-\gamma\left(s_{2}-\bar{s}_{2}\right)<0 \\
\left\{\beta k_{4} \mu_{2}\left(s_{2}\right) x_{2}-\varrho \gamma\left(s_{2}-\bar{s}_{2}\right): \varrho \in[0,1]\right\} \\
\text { if } \beta k_{4} \mu_{2}\left(s_{2}\right) x_{2}-\gamma\left(s_{2}-\bar{s}_{2}\right)=0
\end{array}\right.
\end{aligned}
$$

where $\gamma$ is a positive constant. This multivalued map $K\left(s_{2}, x_{2}, \beta\right)$ is upper semicontinuous with compact and convex values.

We have to point out that the quantity $k_{4} \mu_{2}\left(s_{2}\right) x_{2}$ in (24) and 25) is equal to $Q$ and is on-line measurable.

Theorem 1. For each starting point $\left(s_{2}(0), x_{2}(0), \beta(0)\right)$ from the set $\widetilde{\Omega}_{0}$ with

$$
\widetilde{\Omega}_{0}=\left\{\left(s_{2}, x_{2}, \beta\right) \mid s_{2}>0, x_{2}>0, \beta \in\left(\beta^{-}, \beta^{+}\right)\right\},
$$

there exists a feedback control law $k\left(s_{2}, x_{2}, \beta\right) \in$ $K\left(s_{2}, x_{2}, \beta\right)$ that stabilizes asymptotically the control system (22)-(23) and (24) to $\left(\bar{s}_{2}, \bar{x}_{2}, \bar{\beta}\right)$.

Remark 5. We formulate a stabilization result assuming that the control $u$ is unbounded. In practice, some bounds for the control do exist due to physical evidence. Let us assume that $u \in\left[u_{\min }, u_{\max }\right]$. In the proof presented below, we construct explicitly a Lyapunov function $V$. Assuming that the value of the parameter $k_{4}$ belongs to the interval $\left[k_{4}^{-}, k_{4}^{+}\right]$, an estimate of the constrained stability region of the above controller can be obtained using the level sets of $V$, i.e.,

$$
\Xi=\left\{x \in R^{n}: V(x) \leq c^{\max }\right\},
$$

where $c^{\max }>0$ is the largest number for which every element of $\tilde{\Omega}_{0} \cap \Xi$ is contained in the set

$$
\begin{aligned}
& \Theta=\left\{\left(s_{2}, x_{2}\right): \frac{u_{\min }+\gamma\left(s_{2}-\bar{s}_{2}\right)}{\beta^{+} k_{4}^{+}}\right. \\
&\left.\leq \mu_{2}\left(s_{2}\right) x_{2} \leq \frac{u_{\max }+\gamma\left(s_{2}-\bar{s}_{2}\right)}{\beta^{-} k_{4}^{-}}\right\},
\end{aligned}
$$

consisting of all points that satisfy the control constraints.

Proof. We set

$$
\begin{gathered}
\Omega_{0}=\left\{\left(s_{2}, x_{2}, \beta\right) \mid s_{2}^{i}>s_{2}>0, x_{2}>0,\right. \\
\left.\beta \in\left(\beta^{-}, \beta^{+}\right)\right\} .
\end{gathered}
$$

Since the multivalued map $K\left(s_{2}, x_{2}, \beta\right)$ is upper semicontinuous with compact and convex values, there exist a selection $k\left(s_{2}, x_{2}, \beta\right)$ of the multivalued map $K\left(s_{2}, x_{2}, \beta\right)$ such that the closed-loop system $\Sigma(\omega)$ with exact but unknown values for the model parameters has at last one local solution starting from an arbitrary point of the set $\Omega_{0}$ (cf., for example, the monograph (Filippov, 1988)). Let us note that $\Sigma(\omega)$ is obtained from 22 - 23 and (24) by substituting the control variable $u$ by the feedback $k\left(s_{2}, x_{2}, \beta\right)$. Denote

$k\left(s_{2}, x_{2}, \beta\right)=\beta k_{4} \cdot \mu_{2}\left(s_{2}\right) \cdot x_{2}-\varrho\left(s_{2}, x_{2}, \beta\right) \cdot \gamma \cdot\left(s_{2}-\bar{s}_{2}\right)$,

where $\varrho\left(s_{2}, x_{2}, \beta\right)$ is a constant, equal to 1,0 or $\varrho$ with $\varrho \in[0,1]$, depending on the conditions in the right-hand side branches of (25).

For convenience, we set

$$
\zeta=\left(s_{2}, x_{2}\right), \quad \bar{\zeta}=\left(\bar{s}_{2}, \bar{x}_{2}\right)
$$

One can directly verify that the set $\Omega_{0}$ is strongly invariant with respect to (22)-23) and (24) (Clarke et al., 1998). This means that every trajectory of the closed-loop system $\Sigma(\omega)$ starting from a point $(\zeta, \beta) \in \Omega_{0}$ remains in $\Omega_{0}$. In particular, the coordinates of all points of this trajectory will never vanish. Moreover, if the initial point belongs to the larger set $\widetilde{\Omega}_{0}$, then every trajectory of $\Sigma(\omega)$ starting from a point $(\zeta, \beta) \in \widetilde{\Omega}_{0}$ enters the set $\Omega_{0}$ in finite time. For that reason we can assume without loss of generality that the initial point belongs to the set $\Omega_{0}$.

Using the fact that $s_{2}^{i}=\bar{s}_{2}+\alpha k_{3} \bar{x}_{2}-c_{1}^{*}$, the equations (22) -23) of the closed-loop system $\Sigma(\omega)$ can be written as follows:

$$
\begin{aligned}
\frac{\mathrm{d} s_{2}}{\mathrm{~d} t}= & -k\left(s_{2}, x_{2}, \beta\right) \cdot\left(s_{2}-\bar{s}_{2}+\alpha k_{3}\left(x_{2}-\bar{x}_{2}\right)\right) \\
& -k_{3}\left(\mu_{2}\left(s_{2}\right)-\alpha k\left(s_{2}, x_{2}, \beta\right)\right) \cdot x_{2} \\
\frac{\mathrm{d} x_{2}}{\mathrm{~d} t}= & \left(\mu_{2}\left(s_{2}\right)-\alpha k\left(s_{2}, x_{2}, \beta\right)\right) \cdot x_{2} .
\end{aligned}
$$

Consider the function

$$
\begin{aligned}
& V(\zeta, \beta) \\
& =\left(s_{2}-\bar{s}_{2}+k_{3}\left(x_{2}-\bar{x}_{2}\right)\right)^{2} \\
& +\Gamma\left(\int_{\bar{s}_{2}}^{s_{2}} \frac{v-\bar{s}_{2}}{s_{2}^{i}-v} \mathrm{~d} v+\frac{1}{C} \int_{\bar{\beta}}^{\beta} \frac{w-\bar{\beta}}{\left(w-\beta^{-}\right)\left(\beta^{+}-w\right)} \mathrm{d} w\right)
\end{aligned}
$$

where the parameter $\Gamma>0$ will be determined later. Clearly, the values of this function are nonnegative. If we denote by $F(\zeta, \beta)$ the right-hand side of (26)-27, (24) and take into account the definition of the map $K\left(s_{2}, x_{2}, \beta\right)$ from 25 , then it can be directly checked 
that for each point $(\zeta, \beta)$ of $\Omega_{0}$,

$$
\begin{aligned}
\langle\operatorname{grad} & V(\zeta, \beta), F(\zeta, \beta)\rangle \\
= & -2 k\left(s_{2}, x_{2}, \beta\right)\left(s_{2}-\bar{s}_{2}\right)^{2} \\
& -\left(\Gamma\left(\varrho\left(s_{2}, x_{2}, \beta\right) \gamma+\frac{k_{3} \mu_{2}\left(s_{2}\right) x_{2}}{\left(s_{2}^{i}-s_{2}\right)\left(s_{2}^{i}-\bar{s}_{2}\right)}\right)\right) \\
& \cdot\left(s_{2}-\bar{s}_{2}\right)^{2} \\
& -2(1+\alpha) k_{3} \cdot k\left(s_{2}, x_{2}, \beta\right)\left(s_{2}-\bar{s}_{2}\right)\left(x_{2}-\bar{x}_{2}\right) \\
& -2 \alpha k_{3}^{2} \cdot k\left(s_{2}, x_{2}, \beta\right)\left(x_{2}-\bar{x}_{2}\right)^{2} .
\end{aligned}
$$

The discriminant $D(\zeta, \beta)$ of the last expression, considered as a quadratic function with respect to $s_{2}-\bar{s}_{2}$ and $x_{2}-\bar{x}_{2}$, is

$$
\begin{aligned}
D(\zeta, \beta) & \\
= & -4 k_{3}^{2} \cdot k\left(s_{2}, x_{2}, \beta\right) \cdot \varrho\left(s_{2}, x_{2}, \beta\right) \cdot \gamma \\
& \cdot\left(2 \alpha \cdot \Gamma+(1-\alpha)^{2}\left(s_{2}-\bar{s}_{2}\right)\right) \\
& -4 k_{3}^{2} \cdot k\left(s_{2}, x_{2}, \beta\right) \cdot \mu_{2}\left(s_{2}\right) \cdot x_{2} \\
& \cdot\left(\Gamma \cdot \frac{4 \alpha k_{3}}{\left(s_{2}^{i}-s_{2}\right)\left(s_{2}^{i}-\bar{s}_{2}\right)}-k_{4}(1-\alpha)^{2} \beta\right) .
\end{aligned}
$$

Now we choose the positive parameter $\Gamma$ in such a way that the following inequalities are satisfied:

$$
\begin{array}{r}
2 \alpha \cdot \Gamma+(1-\alpha)^{2}\left(s_{2}-\bar{s}_{2}\right)>0, \\
\Gamma \cdot \frac{4 \alpha k_{3}}{\left(s_{2}^{i}-s_{2}\right)\left(s_{2}^{i}-\bar{s}_{2}\right)}-k_{4}(1-\alpha)^{2} \beta>0 .
\end{array}
$$

Then $D(\zeta, \beta)<0$ is fulfilled for each point $(\zeta, \beta)$ from the set $\Omega_{0}$. Hence,

$$
\langle\operatorname{grad} V(\zeta, \beta), F(\zeta, \beta)\rangle<0
$$

for each point $(\zeta, \beta) \in \Omega_{0} \backslash\{(\bar{\zeta}, \bar{\beta})\}$.

Applying LaSalle's invariance principle (cf., for example, (Khalil, 1992)), it follows that every solution of the system (22)-23), (24) is defined on the interval $[0,+\infty)$ and approaches the largest invariant set of (22)-(23), (24), which is contained in the set $\Omega_{0} \cap$ $\left\{(\zeta, \beta) \in R^{3}: s_{2}=\bar{s}_{2}, \beta \in\left(\beta^{-}, \beta^{+}\right)\right\}$. Taking into account the definition of the multivalued map $K(\cdot)$, it is easy to see that this invariant set consists of the single point $(\bar{\zeta}, \bar{\beta})$. Hence the feedback $k(\cdot)$ stabilizes asymptotically the control system (22)-23), (24) to the point $(\bar{\zeta}, \bar{\beta})$ on the set $\Omega_{0}$.

\section{Adaptive stabilization towards the maximum methane flow rate}

In practice, only the substrate concentrations $s_{2}$ and $s_{2}^{i}$ and the effluent methane flow rate $Q$ are measurable online (cf., for example, (Antonelli et al., 2003)). Consider
Eqn. (5) and let as before $s_{2} \in\left(0, s_{2}^{i}\right)$ be some reference point. Then the function

$$
Q\left(s_{2}\right)=k_{4} \cdot \mu_{2}\left(s_{2}\right) \cdot \frac{s_{2}^{i}+c_{1}^{*}-s_{2}}{\alpha k_{3}},
$$

which is defined on the set of all steady states, has a maximum at a unique point $s_{2}^{\max } \in\left(0, s_{2}^{i}\right)$, that is, $Q_{\max }=$ $Q\left(s_{2}^{\max }\right)$. Denote

$$
u_{\max }=\frac{1}{\alpha} \mu_{2}\left(s_{2}^{\max }\right)
$$

and

$$
x_{2}^{\max }=\frac{s_{2}^{i}+c_{1}^{*}-s_{2}^{\max }}{\alpha k_{3}} .
$$

Our goal now is to stabilize the methanogenic dynamic system (22)-23) towards the (unknown) maximum methane flow rate $Q_{\max }$. For that purpose we write (24) in the form

$$
\frac{\mathrm{d} \beta}{\mathrm{d} t}(t)=-C\left(\beta(t)-\beta^{-}\right)\left(\beta^{+}-\beta(t)\right) Q(t)\left(s_{2}(t)-\bar{s}_{2}\right),
$$

where $Q(t)$ means the methane flow rate measured at the moment of time $t$. We would like to point out that not only $Q(t)$ but all quantities in (28) are on-line measurable. Thus the values of its solution can also be determined online. Since the solution of (28) depends on $\bar{s}_{2}$, we denote it by $\beta_{\bar{s}_{2}}(t), t \in[0,+\infty)$. The last fact allows us to apply on-line the feedback control law

$$
\begin{aligned}
& \left(s_{2}, Q, \beta_{\bar{s}_{2}}\right) \longmapsto k\left(s_{2}, Q, \beta_{\bar{s}_{2}}\right) \\
& \quad=\beta_{\bar{s}_{2}} Q-\varrho\left(s_{2}, Q, \beta_{\bar{s}_{2}}\right) \cdot \gamma \cdot\left(s_{2}-\bar{s}_{2}\right) .
\end{aligned}
$$

According to Theorem 1, this feedback will asymptotically stabilize the control system (22)-(23), (28) to the point $\left(\bar{s}_{2}, \bar{x}_{2}, \bar{\beta}_{\bar{s}_{2}}\right)$ with

$$
\left(\bar{s}_{2}, \bar{x}_{2}, \bar{\beta}_{\bar{s}_{2}}\right)=\left(\bar{s}_{2}, \frac{s_{2}^{i}+c_{1}^{*}-\bar{s}_{2}}{\alpha k_{3}}, \frac{k_{3}}{k_{4}\left(s_{2}^{i}+c_{1}^{*}-\bar{s}_{2}\right)}\right) .
$$

To stabilize the dynamics (22)- 23), (28) by means of the feedback 29], we use the fact that Theorem 1 is valid for any reference point $\bar{s}_{2} \in\left(0, s_{2}^{i}\right)$. We shall construct a sequence of points $\left\{\bar{s}_{2}^{n}\right\}, n=1,2, \ldots$, and use an extremum seeking iterative algorithm to generate a sequence $\left\{Q_{n}\right\}$, which converges to $Q_{\max }$. The algorithm is carried out in two stages: in Stage 1, an interval $[S]=\left[S^{-}, S^{+}\right]$is found such that $\left[S^{-}, S^{+}\right] \subset\left(0, s_{2}^{i}\right)$ and $s_{2}^{\max } \in\left[S^{-}, S^{+}\right]$; in Stage 2, the interval $[S]$ is refined using an elimination procedure based on a Fibonacci search technique (Karmanov, 2000). Stage 2 produces the final interval $\left[S^{*-}, S^{*+}\right]$ such that $\left[S^{*-}, S^{*+}\right] \subseteq\left[S^{-}, S^{+}\right] \subset$ $\left(0, s_{2}^{i}\right), s_{2}^{\max } \in\left[S^{*-}, S^{*+}\right]$ and $S^{*+}-S^{*-} \leq \varepsilon$.

The main steps of the numerical extremum seeking algorithm are presented in Appendix. 
(a)

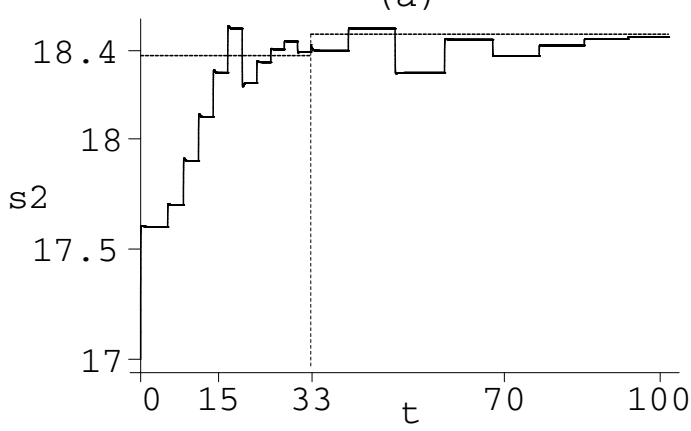

( b)

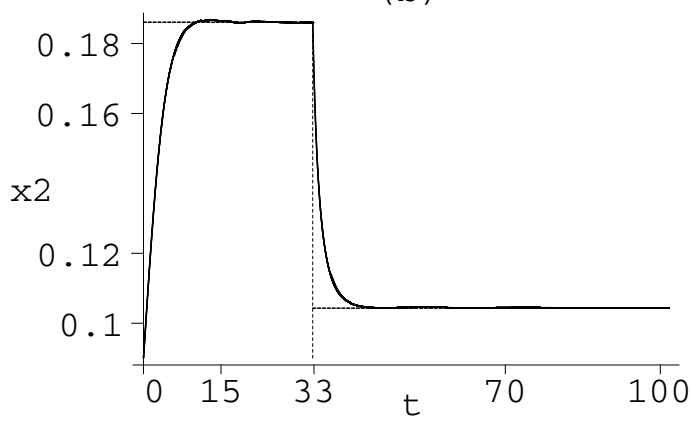

( C)

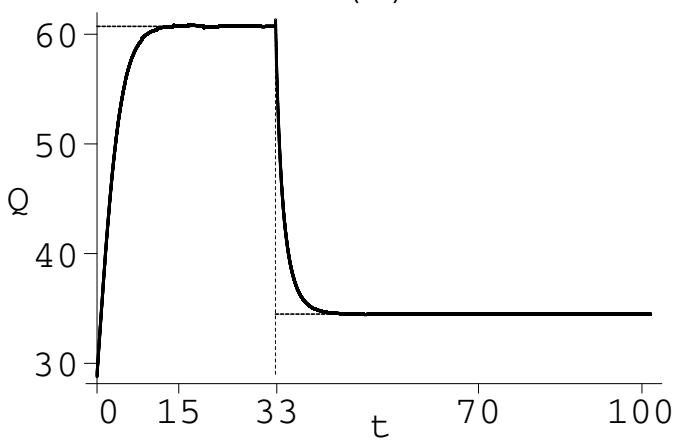

(a)

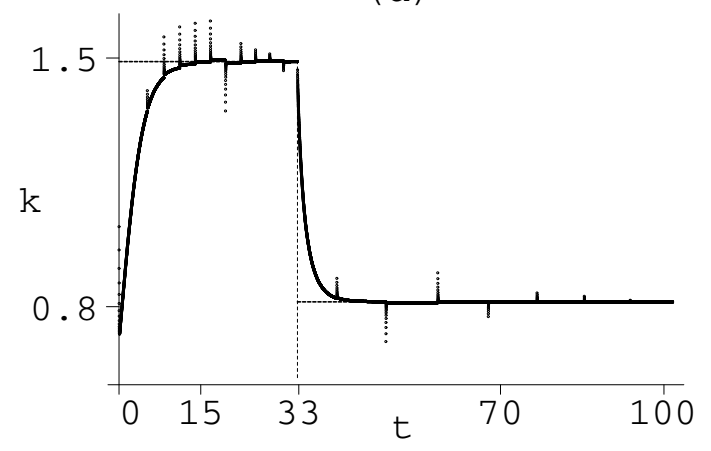

Fig. 2. Time evolution of (a) $s_{2}(t)$, (b) $x_{2}(t)$, (c) $Q(t)$, (d) feedback $k(t)$; horizontal line segments go through $s_{2}^{\max }$, $x_{2}^{\max }, Q_{\max }$ and $u_{\max }$, respectively.

\section{Numerical simulation}

As mentioned before, the exact value of the parameter vector

$$
\omega=\left(\alpha, k_{1}, k_{2}, k_{3}, k_{4}, \mu_{\max }, k_{s_{1}}, \mu_{0}, k_{s_{2}}, k_{I}\right)
$$

is not known. Practical experiments and parameter estimation results (cf. (Alcaraz-González et al., 2002; Grognard and Bernard, 2006; Mailert et al., 2004; Simeonov, 1994; Simeonov, 1999)) give only bounds for the vector components. For that reason, we assume that the model parameters are bounded within compact real intervals $[\alpha],\left[k_{1}\right],\left[k_{2}\right],\left[k_{3}\right],\left[k_{4}\right],\left[\mu_{\max }\right],\left[k_{s_{1}}\right],\left[\mu_{0}\right],\left[k_{s_{2}}\right]$ and $\left[k_{I}\right]$, respectively. Denote by $[\omega]$ the corresponding vector with interval components:

$$
\begin{aligned}
{[\omega]=} & \left([\alpha],\left[k_{1}\right],\left[k_{2}\right],\left[k_{3}\right],\left[k_{4}\right],\left[\mu_{\max }\right],\left[k_{s_{1}}\right],\right. \\
& {\left.\left[\mu_{0}\right],\left[k_{s_{2}}\right],\left[k_{I}\right]\right) . }
\end{aligned}
$$

Using the intervals for the parameters, we can find the bounds $\beta^{-}$and $\beta^{+}$for $\bar{\beta}$ explicitly,

$$
\beta^{-}=\frac{k_{3}^{-}}{k_{4}^{+}\left(s_{2}^{i}+c_{1}^{*}-\bar{s}_{2}\right)}, \quad \beta^{+}=\frac{k_{3}^{+}}{k_{4}^{-}\left(s_{2}^{i}+c_{1}^{*}-\bar{s}_{2}\right)}
$$

for any reference point $\bar{s}_{2}$. In the extremum seeking algorithm these bounds are computed for every choice of the reference point $\bar{s}_{2}$.

In the numerical simulations we consider the following intervals for the coefficients

$$
\begin{aligned}
{[\alpha] } & =[0.3,0.6], & {\left[k_{1}\right] } & =[9.5,11.5], \\
{\left[k_{2}\right] } & =[27.6,29.6], & {\left[k_{3}\right] } & =[1064,1084], \\
{\left[k_{4}\right] } & =[650,700], & {\left[\mu_{\max }\right] } & =[1,1.4], \\
{\left[k_{s_{1}}\right] } & =[6.5,7.9], & {\left[\mu_{0}\right] } & =[0.64,0.84], \\
{\left[k_{s_{2}}\right] } & =[8.28,10.28], & {\left[k_{I}\right] } & =[15,17] .
\end{aligned}
$$

These intervals are chosen to enclose experimentally validated coefficient values, see e.g., (Alcaraz-González et al., 2002; Antonelli et al., 2003).

One can easily see that for any choice of $\omega \in[\omega]$ the relation $u_{2}<u_{1}$ holds true (see Remark 4).

The input concentrations $s_{1}^{i}$ and $s_{2}^{i}$ are assumed to be constant, $s_{1}^{i}=7, s_{2}^{i}=70$. Further, we take $\bar{s}_{1}=1.4$.

To demonstrate the robustness of the feedback with respect to the model uncertainties we proceed as follows: At the initial moment $\left(t_{0}=0\right)$, we choose random values for the model parameters from the corresponding intervals and consider them as the "exact" vector $\omega$. These values are kept constant until the system stabilizes to $Q_{\max }$. Then, at some time moment $t=t_{1}>t_{0}$, another set of random values for the model parameters is chosen to represent again the "exact" vector $\omega$. The process is repeated. Thereby the last computed values for $s_{2}, x_{2}$ and $\beta$ are considered as new initial conditions. 
During algorithm execution, all intermediate numerical values for $s_{2}, x_{2}, Q$ and the feedback $k$ were collected in arrays and then plotted to visualize the results. Figure 2 shows the time profiles of the state variables $s_{2}(t)$ and $x_{2}(t)$ (plots (a) and (b), respectively), of $Q(t)$ (plot (c)) and of the feedback $k(t)$ (plot (d)). In the plots the horizontal dash-line segments go through $s_{2}^{\max }, x_{2}^{\max }, Q_{\max }$ and $u_{\max }$, respectively. The vertical line segments mark the moment of time $t_{1}$, where the coefficients are randomly changed.

The numerical simulations were carried out in the computer algebra system Maple. Thereby we used symbolic manipulations to find steady states, an ODE solver together with the implemented extremum seeking algorithm and graphic visualization facilities to plot the numerical results.

\section{Conclusion}

In the paper we studied a four-dimensional nonlinear dynamic system which models a biological two-stage wastewater treatment process. It was shown that the openloop system undergoes local transcritical bifurcations of the steady states when the control parameter (the dilution rate) $u$ takes different admissible values. Assuming that the acidogenesis (first stage) had been already stabilized to some operating point $s_{1}^{*}$, a nonlinear adaptive feedback was proposed, which stabilizes asymptotically the closedloop second stage dynamics (the methanogenic phase) towards the maximum methane production rate $Q_{\max }$. For that purpose, we first showed that for any previously chosen reference point $\bar{s}_{2}$ we can asymptotically stabilize the model to an equilibrium point $\bar{\zeta}=\left(\bar{s}_{2}, \bar{x}_{2}\right)$, whose projection on the $s_{2}$-axis is $\bar{s}_{2}$. It should be pointed out that if the control input $u$ is unbounded, then stabilization is global in the sense that the starting point can be any point from the unbounded set $\widetilde{\Omega}_{0}$. Further, a numerical extremum seeking algorithm was used to stabilize the closed-loop system into an interval $\left[S^{*}\right]$, containing the equilibrium point $s_{2}^{\max }$ for which the methane output flow rate $Q$ takes its maximum $Q_{\max }$. The interval $\left[S^{*}\right]$ can be made as tight as desired depending on a constant $\varepsilon>0$, which has to be celebrated by the user. Assuming that the model parameters are unknown but bounded within compact intervals, numerical experiments were carried out to demonstrate the robustness of the proposed control law. Our further efforts will be directed to designing an adaptive feedback law for asymptotic stabilization of the whole four-dimensional dynamics towards some reference point; this feedback law should depend on on-line measurable model quantities and be robust with respect to model uncertainties.

\section{Acknowledgment}

This work has been partially supported by the Bulgarian Science Fund under Grant No. DO 02-359/2008. The authors are grateful to the anonymous referees for the valuable advices and comments.

\section{References}

Alcaraz-González, V., Harmand, J., Rapaport, A., Steyer, J.-P. and Pelayo-Ortiz, C. (2002). Software sensors for highly uncertain WWTPs: A new apprach based on interval observers, Water Research 36(10): 2515-2524.

Antonelli, R., Harmand, J., Steyer, J.-P. and Astolfi, A. (2003). Set-point regulation of an anaerobic digestion process with bounded output feedback, IEEE Transactions on Control Systems Technology 11(4): 495-504.

Bastin, G. and Dochain, D. (1990). On-line Estimation and Adaptive Control of Bioreactors, Elsevier Science, New York, NY.

Bernard, O., Hadj-Sadok, Z. and Dochain, D. (2000). Advanced monitoring and control of anaerobic wastewater treatment plants: Dynamic model development and identification, Proceedings of the 5th IWA International Symposium WATERMATEX 2000, Gent, Belgium, pp. 3.57-3.64.

Bernard, O., Hadj-Sadok, Z., Dochain, D., Genovesi, A. and Steyer, J.-P. (2001). Dynamical model development and parameter identification for an anaerobic wastewater treatment process, Biotechnology and Bioengineering 75(4): 424-438.

Carr, J. (1981). Applications of Centre Manifold Theory, Applied Mathematical Science, Vol. 35, Springer, New York, NY, Heidelberg/Berlin.

Clarke, F., Ledyaev, Yu., Stern, R. and Wolenski P. (1998). Nonsmooth Analysis and Control Theory, Graduate Text in Mathematics, Vol. 178, Springer, Berlin.

Dimitrova, N. and Krastanov, M. I. (2006). Nonlinear adaptive control of an uncertain wastewater treatment model, IEEE Proceedings of the 12th GAMM-IMACS International Symposium on Scientific Computing, Computer Arithmetic and Validated Numerics, Duisburg, Germany, p.11.

Filippov, A. F. (1988). Differential Equations with Discontinuous Right-hand Sides, Mathematics and Its Applications: Soviet Series, Vol. 18, Kluwer Academic Publishers, Dordrecht.

Grognard, F. and Bernard, O. (2006). Stability analysis of a wastewater treatment plant with saturated control, Water Science Technology 53(1): 149-157.

Harmand, J., Rapaport, A. and Trofino, A. (2003). Optimal design of a series of two reactors-Some new results, American Institute of Chemical Engineering Journal 49(6): 1433-1450.

Heinzle, E., Dunn, I. J. and Ryhiner, G. B. (1993). Modelling and control for anaerobic wastewater treatment, Advances in Biochemical Engineering and Biotechnology 48: 79114. 
Hess, J. and Bernard, O. (2008). Design and study of a risk management criterion for an unstable anaerobic wastewater treatment process, Journal of Process Control 18(1):7179.

Karmanov, V. (2000). Mathematical Programming, FIZMATLIT, Moscow, (in Russian).

Khalil, H. K. (1992). Nonlinear Systems, Macmillan Publishing Company, New York, NY.

Maillert, L., Bernard, O. and Steyer, J.-P. (2004). Nonlinear adaptive control for bioreactors with unknown kinetics, Automatica 40(8): 1379-1385.

Marcos, N. I., Guay, M., Dochain, D. and Zhang, T. (2004). Adaptive extremum-seeking control of a continuous stirred tank bioreactor with Haldane's kinetics, Journal of Process Control 14(3): 317-328.

Schoefs, O., Dochain, D., Fibrianto, H. and Steyer, J.-P. (2003). Modelling and identification of a distributedparameter system for an anaerobic wastewater treatment process, Chemical Engineering Research and Design 81(A9): 1279-1288.

Simeonov, I. (1994). Modelling and control of anaerobic digestion of organic waste, Chemical and Biochemical Engineering Quaterly 8(2): 45-52.

Simeonov, I. (1999). Mathematical modelling and parameter estimation of anaerobic fermentation processes, Bioprocess Engineering 21(4): 377-381.

Simeonov, I., Noykova, N. and Stoyanov, S. (2004). Modelling and extremum seeking control of the anaerobic digestion, Proceedings of the International IFAC Workshop DECOMTT, Bansko, Bulgaria, pp. 289-294.

Simeonov, I., Noykova, N. and Gyllenberg, M. (2007). Identification and extremum seeking control of the anaerobic digestion of organic wastes, Cybernetics and Information Technologies 7(2): 73-84.

Wang, H.-H., Krstic, M. and Bastin, G. (1999). Optimizing bioreactors by extremum seeking, International Journal of Adaptive Control and Signal Processing 13(8): 651-669.

Wiggins, S. (1990). Introduction to Applied Nonlinear Dynamical Systems and Chaos, Springer, New York, NY.

\section{Appendix}

Below we present the main steps of the numerical extremum seeking algorithm. The steps are executed in the given order except as indicated by branching. We assume tolerances $\varepsilon>0, h>0$ and $\varepsilon_{s}>0$ to be given.

\section{Extremum Seeking Algorithm}

Stage 1. Determine an interval $[S]=\left[S^{-}, S^{+}\right]$such that $[S] \subset\left(0, s_{2}^{i}\right]$ and $s_{2}^{\max } \in[S]$.

Step 1.0. Choose $\bar{s}_{2}^{0} \in\left(0, s_{2}^{i}\right)$. Apply the feedback $k\left(s_{2}, Q, \beta_{\bar{s}_{2}^{0}}\right)$ to stabilize the system to $\bar{s}_{2}^{0}$. According to Theorem 1 , there exists a moment of time $t_{0}>0$ such that $\left|s_{2}\left(t_{0}\right)-\bar{s}_{2}^{0}\right|<\varepsilon_{s}$; set $\bar{s}_{2}^{0}:=s_{2}\left(t_{0}\right), Q_{0}:=Q\left(t_{0}\right)$.
Step 1.1. Set $\sigma:=1, \bar{s}_{2}^{1}:=\bar{s}_{2}^{0}+\sigma h$. Apply the feedback $k\left(s_{2}, Q, \beta_{\bar{s}_{2}^{1}}\right)$ to stabilize the system to $\bar{s}_{2}^{1}$. According to Theorem 1 , there exists a moment of time $t_{1}>0$ such that $\left|s_{2}\left(t_{1}\right)-\bar{s}_{2}^{1}\right|<\varepsilon_{s}$; set $\bar{s}_{2}^{1}:=s_{2}\left(t_{1}\right), Q_{1}:=Q\left(t_{1}\right)$. If $Q_{1}>Q_{0}$ then go to Step 1.3 else go to Step 1.2.

Step 1.2. Set $\sigma:=-1, \bar{s}_{2}^{1}:=\bar{s}_{2}^{0}+\sigma h$. Apply the feedback $k\left(s_{2}, Q, \beta_{\bar{s}_{2}^{1}}\right)$ to stabilize the system to $\bar{s}_{2}^{1}$. According to Theorem 1, there exists a moment of time $t_{1}>0$ such that $\left|s_{2}\left(t_{1}\right)-\bar{s}_{2}^{1}\right|<\varepsilon_{s}$; set $\bar{s}_{2}^{1}:=s_{2}\left(t_{1}\right), Q_{1}:=Q\left(t_{1}\right)$.

If $Q_{1}>Q_{0}$ then go to Step 1.3

If $Q_{1} \leq Q_{0}$ then set $h:=h / 2$;

if $h \leq \varepsilon / 2$ then set $\left[S^{*}\right]:=\left[\bar{s}_{2}^{0}-\varepsilon, \bar{s}_{2}^{0}+\varepsilon\right]$ and stop computations without going to Stage 2;

if $h>\varepsilon / 2$ then go to Step 1.1.

Step 1.3. Set $h:=2 h, \bar{s}_{2}^{2}:=\bar{s}_{2}^{1}+\sigma h$. Apply the feedback $k\left(s_{2}, Q, \beta_{\bar{s}_{2}^{2}}\right)$ to stabilize the system to $\bar{s}_{2}^{2}$. According to Theorem 1, there exists a moment of time $t_{2}>0$ such that $\left|s_{2}\left(t_{2}\right)-\bar{s}_{2}^{2}\right|<\varepsilon_{s}$; set $\bar{s}_{2}^{2}:=s_{2}\left(t_{2}\right), Q_{2}:=Q\left(t_{2}\right)$.

If $Q_{2} \leq Q_{1}$ then set $[S]=\left[S^{-}, S^{+}\right]:=\left[\bar{s}_{2}^{0}, \bar{s}_{2}^{2}\right]$ and go to Stage 2 .

If $Q_{2}>Q_{1}$ then set $\bar{s}_{2}^{0}:=\bar{s}_{2}^{1}, \bar{s}_{2}^{1}:=\bar{s}_{2}^{2}, Q_{1}:=$ $Q_{2}$; repeat Step 1.3.

Stage 2. Starting with $[S]=\left[S^{-}, S^{+}\right]$, determine an interval $\left[S^{*}\right]=\left[S^{*-}, S^{*+}\right]$ with $s_{2}^{\max } \in\left[S^{*}\right]$ and $S^{*+}-$ $S^{*-} \leq \varepsilon$.

Denote $s_{2}^{0^{-}}:=S^{-}, s_{2}^{0^{+}}:=S^{+}, \lambda:=(\sqrt{5}-1) / 2$; compute $\Delta_{1}:=s_{2}^{0^{+}}-s_{2}^{0^{-}}$.

Step 2.0. Compute $\Delta_{2}:=(1-\lambda) \Delta_{1}, p_{0}:=s_{2}^{0^{-}}+\Delta_{2}$, $q_{0}:=s_{2}^{0^{+}}-\Delta_{2}$.

Step 2.1. Apply the feedback $k\left(s_{2}, Q, \beta_{p_{0}}\right)$ to stabilize the system to $p_{0}$. According to Theorem 1, there exists a moment of time $t_{p_{0}}>0$ such that $\left|s_{2}\left(t_{p_{0}}\right)-p_{0}\right|<\varepsilon_{s}$; set $p_{0}:=s_{2}\left(t_{p_{0}}\right)$, $Q_{p_{0}}:=Q\left(t_{p_{0}}\right)$

Apply the feedback $k\left(s_{2}, Q, \beta_{q_{0}}\right)$ to stabilize the system to $q_{0}$. According to Theorem 1, there exists a moment of time $t_{q_{0}}>0$ such that $\left|s_{2}\left(t_{q_{0}}\right)-q_{0}\right|<\varepsilon_{s}$; set $q_{0}:=s_{2}\left(t_{q_{0}}\right)$, $Q_{q_{0}}:=Q\left(t_{q_{0}}\right)$.

Step 2.2. Set $\Delta_{3}:=q_{0}-p_{0}$. If $Q_{p_{0}}>Q_{q_{0}}$ then set $s_{2}^{1^{-}}:=s_{2}^{0^{-}}, s_{2}^{1^{+}}:=q_{0}$, $p_{1}:=s_{2}^{1^{-}}+\Delta_{3}, q_{1}:=p_{0}$; If $Q_{p_{0}} \leq Q_{q_{0}}$ then set $s_{2}^{1^{-}}:=p_{0}, s_{2}^{1^{+}}:=s_{0}^{+}$, $p_{1}:=q_{0}, q_{1}:=s_{2}^{1^{+}}-\Delta_{3}$.

Compute $\Delta_{1}:=s_{2}^{1^{+}}-s_{2}^{1^{-}}$. 
Step 2.3. If $\Delta_{1} \leq \varepsilon$ then set $\left[S^{*}\right]:=\left[s_{2}^{1^{-}}, s_{2}^{1^{+}}\right]$ and stop computations.

If $\Delta_{1}>\varepsilon$ then

if $p_{1} \geq q_{1}$ then set $s_{2}^{0^{-}}:=s_{2}^{1^{-}}, s_{2}^{0^{+}}:=s_{2}^{1^{+}}$ and go to Step 2.0.

if $p_{1}<q_{1}$ then

if $Q_{p_{0}}>Q_{q_{0}}$ then apply the feedback $k\left(s_{2}, Q, \beta_{p_{1}}\right)$ to stabilize the system to $p_{1}$. According to Theorem 1, there exists a moment of time $t_{p_{1}}>0$ such that $\mid s_{2}\left(t_{p_{1}}\right)-$ $p_{1} \mid<\varepsilon_{s}$; set $p_{1}:=s_{2}\left(t_{p_{1}}\right), Q_{p_{1}}:=$ $Q\left(t_{p_{1}}\right)$

if $Q_{p_{0}} \leq Q_{q_{0}}$ then apply the feedback $k\left(s_{2}, Q, \beta_{q_{1}}\right)$ to stabilize the system to $q_{1}$. According to Theorem 1, there exists a moment of time $t_{q_{1}}>0$ such that $\mid s_{2}\left(t_{q_{1}}\right)-$ $q_{1} \mid<\varepsilon_{s} ;$ set $q_{1}:=s_{2}\left(t_{q_{1}}\right), Q_{q_{1}}:=$ $Q\left(t_{q_{1}}\right)$.

Set $p_{0}:=p_{1}, q_{0}:=q_{1}, s_{2}^{0^{-}}:=s_{2}^{1^{-}}$, $s_{2}^{0^{+}}:=s_{2}^{1^{+}}, Q_{p_{0}}:=Q_{p_{1}}, Q_{q_{0}}:=Q_{q_{1}}$; go to Step 2.2.

\section{Comments on the Algorithm}

1. The algorithm works on-line. Having determined the desired interval $\left[S^{*}\right]$, we set $\bar{s}_{2}:=\left(S^{*-}+S^{*+}\right) / 2$ and stabilize the dynamic system towards it. The computational process continues to work without any changes until the system changes due to parameter perturbations. Then the algorithm starts to work either from Stage 1 or from Stage 2 depending on whether $\bar{s}_{2}$ belongs to $[S]$ or $\left[S^{*}\right]$.
2. At any step of the algorithm, the last computed values for $s_{2}$ and $x_{2}$ are used as initial conditions for the next step. For $\beta$, the last computed value is checked whether $\beta \in\left(\beta^{-}, \beta^{+}\right)$; if not, then it is changed to $\beta=\left(\beta^{-}+\right.$ $\left.\beta^{+}\right) / 2$.

3. The algorithm may terminate at Step 1.2 without going to Stage 2 only in the case when the current set point $\bar{s}_{2}$ is sufficiently close to the maximum point $s_{2}^{\max }$, that is if $\left|\bar{s}_{2}-s_{2}^{\max }\right| \leq \varepsilon$.

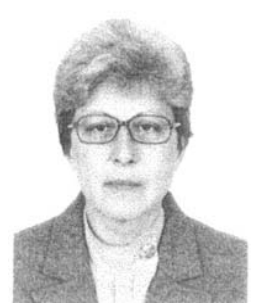

Neli Dimitrova received the M.Sc. and $\mathrm{Ph} . \mathrm{D}$. degrees in mathematics from the University of Sofia, Bulgaria, in 1977 and 1997, respectively. She is currently an associate professor at the Institute of Mathematics and Informatics of the Bulgarian Academy of Sciences. Her areas of interests are numerical analysis, dynamical systems, and applications to mathematical modeling.

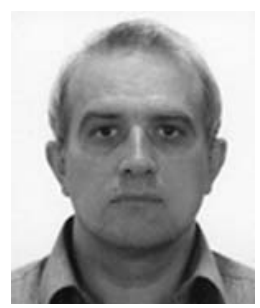

Mikhail Krastanov received the M.Sc. and Ph.D. degrees in mathematics from the University of Sofia, Bulgaria, in 1983 and 1989, respectively. He is currently an associate professor at the Institute of Mathematics and Informatics of the Bulgarian Academy of Sciences. His areas of interests are nonsmooth and setvalued analysis, geometrical methods in control theory, and applications of system theory.

Received: 11 September 2008

Revised: 9 February 2009 